\title{
Effect of climate warming on the timing of autumn leaf senescence reverses at the summer solstice
}

Constantin Zohner ( $\nabla$ constantin.zohner@gmail.com )

ETH Zurich https://orcid.org/0000-0002-8302-4854

\section{Leila Mirzagholi}

ETH Zurich

\section{Susanne Renner}

Washington University

\section{Lidong Mo}

ETH Zurich https://orcid.org/0000-0003-3805-7638

\section{Raymo Bucher}

ETH Zurich

\section{Daniel Palouš}

Charles University in Prague

\section{Yann Vitasse}

University of Neuchâtel https://orcid.org/0000-0002-7454-505X

\section{Yongshuo Fu}

Beijing Normal University

\section{Benjamin Stocker}

ETH

\section{Thomas Crowther}

Swiss Federal Institute of Technology in Zurich https://orcid.org/0000-0001-5674-8913

\section{Biological Sciences - Article}

Keywords: Plant growth, Senescence, Phenology, Global warming, Climate change, Carbon cycle

Posted Date: February 9th, 2022

DOI: https://doi.org/10.21203/rs.3.rs-1336514/v1

License: (9) This work is licensed under a Creative Commons Attribution 4.0 International License. Read Full License 
Constantin M. Zohner ${ }^{1 *}$, Leila Mirzagholi', Susanne S. Renner ${ }^{2}$, Lidong Mo ${ }^{1}$, Raymo Bucher ${ }^{1}$, Daniel ${ }^{1}$ Institute of Integrative Biology, ETH Zurich (Swiss Federal Institute of Technology), Universitätsstrasse 16, States; ${ }^{3}$ Department of Experimental Plant Biology, Charles University in Prague, Viničná 5, CZ 12844 , Czech Republic; ${ }^{4}$ WSL Swiss Federal Institute for Forest, Snow and Landscape Research, Birmensdorf, Switzerland; ${ }^{5}$ College of Water Sciences, Beijing Normal University, Beijing, China; ${ }^{6 D e p a r t m e n t}$ of Environmental Systems Science, Institute of Agricultural Sciences, ETH Zurich, Switzerland.

*Author for correspondence: Constantin Zohner, constantin.zohner@gmail.com

\section{Keywords:}

Plant growth I Senescence I Phenology I Global warming I Climate change I Carbon cycle 
25

26

27

28

29

30

31

32

33

34

35

36

37

38

39

40

41

42

43

44

45

46

47

48

49

50

51

52

53

54

55

56

57

58

59

60

61

62

63

64

65

66

67

68

69

70

71

72

73

74

75

76

77

78

\section{Abstract}

Climate change is causing shifts in the growing seasons of plants ${ }^{1,2}$, affecting species performance and interactions ${ }^{3,4}$ as well as global carbon, water and nutrient cycles, 5 . How the timing of autumn leaf senescence in extra-tropical forests will change remains unclear because of the complex seasonal interaction of climate warming, earlier and enhanced vegetation activity, and the annual day-length cycle ${ }^{7-}$ 10. Here we use experiments, long-term ground observations, and satellite-based vegetation monitoring to show that early-season and late-season warming have opposite effects on the onset and progression of leaf senescence, with a reversal occurring at the year's longest day (summer solstice). Across $\sim 84 \%$ of the northern forest area, increased temperature and vegetation activity before the solstice led to an earlier senescence onset ( $10 \%$ greenness loss) of, on average, $-1.6 \pm 0.1$ days-per- ${ }^{\circ} \mathrm{C}$, while warmer post-solstice temperatures did not affect senescence onset but reduced its speed (progression to $50 \%$ greenness loss) by $0.8 \pm 0.1$ days-per- ${ }^{\circ} \mathrm{C}$. Due to the earlier senescence onset, the day at which autumn temperature starts driving senescence progression has been shifting to ever earlier dates, between 1951-2015 at a rate of $0.20 \pm 0.07$ days per year. These developmental constraints suggest that senescence will start earlier but progress more slowly in the future, revealing Northern Hemisphere-wide compensation effects on trends in growing-season length, caused by enhanced pre-solstice vegetation activity. This new mechanistic insight improves our ability to model carbon uptake by extra-tropical forests under climate change.

\section{Main text}

The phenological cycles of trees exert a strong control on the structure and functioning of ecosystems 4,11 and global carbon, water and nutrient cycles $2,5,6$. Anthropogenic climate change has resulted in shifts in the growing seasons of temperate and boreal trees, with the start of the season today occurring, on average, two weeks earlier than it did during the $19^{\text {th }}$ and $20^{\text {th }}$ century ${ }^{12}$ and the end of the season (EOS) being delayed ${ }^{1,6,13}$. Each day of a longer growing season may increase net ecosystem carbon uptake by 3.0-9.8 $\mathrm{gC} \mathrm{m}^{-2}\left(\mathrm{ref}^{6}\right)$. Yet, due to the complex and interactive effects of growing-season climate and the annual daylength cycle the direction of EOS changes in response to climate change has remained highly uncertain $8,9,14,15$.

Characterizing the interplay among the environmental drivers of EOS is integral to improving our understanding of vegetation development and tree growth. A key outstanding question is how day length and climate effects interact and vary throughout the growing season. Local observations and experiments have shown that early-season warming, causing earlier spring leaf-out and faster development, tends to advance EOS dates ${ }^{8-10,16}$, whereas late-season warming has the opposite effect, delaying the EOS 17,18 . Accelerated development and increased vegetation activity in the beginning of the season might drive earlier autumn senescence through a variety of possible mechanisms, such as developmental and nutrient constraints $^{9,19}$, seasonal built-up of water stress 20,21 , or radiation-induced leaf ageing 22 . In contrast, later on in the season, a direct effect of temperature (cooling) is likely to dominate phenological responses ${ }^{14,23,24}$. If these trends are correct, then climate warming has opposing effects at the start and end of the growing season, with a reversal of effects somewhere in between. Yet, we lack a global understanding of the seasonal effects of plant growth and climate on autumn phenology, limiting our ability to forecast future growing seasons and carbon uptake in extra-tropical forests.

Here we test whether early-season vegetation activity and development drive an earlier EOS across northern forests, with day length providing the linkage between seasonal activity, air temperature warming, and autumn phenology (Fig. 1). Cell division, tissue formation, and growth in northern trees are highest at the beginning of the season and decline with shortening days $25-28$, the adaptive reason being the limited time remaining for tissue maturation and bud set before the first frost ${ }^{29}$. We thus expected that inter-annual variation in EOS timing is a function of early-season growth, with an earlier onset of senescence in warm, high-activity years than in cold, low-activity years (Fig. 1 scenario 1 versus 2). Once EOS has begun, its progression (towards full dormancy) should be modulated by autumn temperature, with faster chlorophyll breakdown in colder autumns than in warmer autumns ${ }^{24}$ (Fig. 1 scenario A versus B). If early-season development continues to accelerate due to advances in leaf-out timing and higher air temperature 2,6,30,31, this might cause an ever earlier EOS onset, eventually outweighing the delay in the progression of senescence due to warmer autumns. 
101

102

103

104

105

106

107

108

109

110

111

112

113

114

115

116

117

118

119

120

121

122

123

124

125

126

127

128

129

130

131

132

To test these hypotheses, we combined phenology data from i) satellite observations across Northern Hemisphere temperate and boreal forests, ii) ground-sourced European observations from widespread deciduous trees ${ }^{32}$, and iii) controlled experiments on European beech. As a proxy for vegetation activity, we used three photosynthesis models (satellite-derived gross-primary productivity [GPP] ${ }^{33}$, LPJ9 and $\mathrm{p}$ model ${ }^{34}$ ). We then ran linear models to test for the monthly and seasonal effects of photosynthesis, temperature, short-wave radiation and water availability on EOS dates. The satellite data allowed us to differentiate between the onset of senescence and its progression, by analysing the dates when greenness had dropped by $10 \%\left(E O S_{10}\right)$ or $50 \%\left(E S_{50}\right)$ relative to the seasonal maximum. The experiments allowed us to directly test for seasonal variation in the effects of day length, air temperature, radiation, water, and nutrient availability. Finally, we mapped the relative effects of early-season vegetation activity and lateseason climate across Northern Hemisphere temperate and boreal forests to test for possible historicbiogeographic patterns in the drivers of autumn senescence.

Satellite-based phenology data (Fig. 2), European plot data (Fig. 3), and experiments (Fig. 4) all revealed a consistent advancing effect of pre-solstice (i.e., pre-21 June) vegetation activity on EOS dates, which declined after the June solstice. Thus, across $~ 84 \%$ of the northern forest area (32\% or $22 \%$ with $p$ $<0.1$ or $<0.05$ ), increased pre-solstice photosynthesis and temperature led to an earlier onset of senescence (Fig. 2a,c and Extended Data Fig. 1a,c), with each 10\% increase in photosynthesis and each $1^{\circ} \mathrm{C}$ increase in temperature on average resulting in $-2.8 \pm 0.1$ and $-1.6 \pm 0.1$ days earlier $\mathrm{EOS}_{10}$, respectively (Fig. $2 \mathrm{i}$ and Extended Data Fig. 1i). The advancing effect of pre-solstice vegetation activity was most pronounced at mid-latitudes $\left(45-60^{\circ} \mathrm{N}\right)$ and decreased toward lower latitudes (Fig. 2b). A significant delaying effect of pre-solstice activity was found for fewer than $1 \%$ of pixels (Fig. $2 \mathrm{~d}, \mathrm{e}$ ). Increased post-solstice photosynthesis and temperature, by contrast, had a much smaller effect on the onset of senescence $\left(E^{2} \mathrm{~S}_{10}\right.$ dates; Figs. $2 f-h$ and $5 \mathrm{a}$ and Extended Data Fig. 1f- $\mathrm{h}$ ) but slowed down its progression (EOS ${ }_{50}$ dates) by, on average, $0.8 \pm 0.1$ days per ${ }^{\circ} \mathrm{C}$ (see Figs. $5 \mathrm{~b}$ and Extended Data Fig. 2 for satellite observations and Figs. 3 and $5 \mathrm{c}$ for European plots). Precipitation and $\mathrm{CO}_{2}$ levels had comparatively small effects (Fig. 2f).

The reversal at the summer solstice of the effects that air temperature and vegetation activity have on EOS dates was consistent across i) both EOS metrics used here, i.e., the onset of senescence (EOS ${ }_{10}$; Extended Data Fig. 1) and its progression (EOS ${ }_{50}$; Extended Data Fig. 2), ii) forest types (Fig. 2a and Extended Data Fig. 3), and iii) a set of alternative variables linked to growing-season activity and development, namely climate-derived productivity (Figs. S1 and S2), day-time temperature ( $T_{\text {day; }}$; Extended Data Figs. 1 and 2), and short-wave radiation (Figs. S3 and S4). The effect reversal at the June solstice was further supported by an analysis that used 10-day moving steps around the solstice (Fig. 2h). Furthermore, along the full latitudinal gradient $\left(30^{\circ} \mathrm{N}-65^{\circ} \mathrm{N}\right)$ studied here, the early-season period during which vegetation activity exhibited an advancing effect on $\mathrm{EOS}_{10}$ dates consistently ended right after the solstice at June 26 (Extended Data Fig. 4).

Our finding that the onset of senescence is driven by pre-solstice activity and development, while its rapid or slow progression depends on autumn temperature (Fig. 5b,c and Extended Data Fig. 2) suggests that, under global warming, senescence will start earlier but progress more slowly (scenario 2B in Fig. 1). Indeed, analysis of the temporal trends in remotely-sensed EOS dates and pre-solstice vegetation activity showed that across all analysed northern forest pixels, the onset of senescence (EOS 10 date) has advanced by an average of $-0.4 \pm 0.1$ days per decade between 2001-2018 (Fig. 2j), in parallel with increased presolstice vegetation activity (Extended Data Fig. 5a-b), with the strongest advances in EOS $_{10}$ dates found for regions with the largest increase in pre-solstice GPP (Extended Data Fig. 5k). By contrast, senescence progression ( $E S_{50}$ date) has slowed by an average of $1.5 \pm 0.1$ days per decade (Extended Data Fig. 2l), in parallel with warmer autumns (Extended Data Fig. 5i,j), with the largest extension of the overall senescence duration found for regions with the largest increases in autumn temperature (Extended Data Fig. $5 \mathrm{~m}$ ). When removing the effect of pre-solstice photosynthesis (Fig. 2i) by including it as a fixed effect in addition to year, the model predicted delays in $\mathrm{EOS}_{10}$ and $\mathrm{EOS}_{50}$ dates of $+1.3 \pm 0.1$ and $+2.0 \pm 0.1$ days per decade, respectively (Figs. $2 \mathrm{k}$ and $\mathrm{S} 2 \mathrm{~m}$ ), underscoring the strong advancing effect of the ongoing acceleration in pre-solstice vegetation activity on temporal trends in leaf senescence.

In line with the satellite observations, high pre-solstice vegetation activity also correlated with advanced $\mathrm{EOS}_{50}$ dates in the European plot data, across all four species (Fig. 3b) and across a set of alternative variables (LPJ model-derived photosynthesis [Fig. 3], P-model-derived photosynthesis [Fig. S5], day-time temperature [ $\mathrm{T}_{\text {day }}$; Fig. S6], and cumulative short-wave radiation [Fig. S7]. Based on these findings, 
133

134

135

136

137

138

139

140

141

142

143

144

145

146

147

148

149

150

151

152

153

154

155

156

157

158

159

160

161

162

163

164

165

166

167

168

169

170

171

172

173

174

175

176

177

178

179

180

181

182

183

184

185 we ran multivariate mixed models, including or excluding the effects of pre-solstice or post-solstice (solstice to mean $\mathrm{EOS}_{50}$ ) activity and precipitation to determine the relative importance of seasonal activity, precipitation, $\mathrm{CO}_{2}$ levels, and autumn night-time temperature (Autumn $\mathrm{T}_{\text {night }}$ ). Pre-solstice activity and autumn $\mathrm{T}_{\text {night }}$ had the strongest effects on $\mathrm{EOS}_{50}$ dates, with the effect of pre-solstice photosynthesis being $\sim 3$ times greater than that of precipitation and atmospheric $\mathrm{CO}_{2}$ (Fig. 3h). EOS predictions from these models show that the model representing both pre- and post-solstice effects adequately captures withinsite $\mathrm{EOS}_{50}$ trends in response to mean annual temperature (advance of -0.4 days per each ${ }^{\circ} \mathrm{C}$ increase in mean annual temperature, Fig. $3 \mathrm{~g}$ ). In contrast, the post-solstice model representing only post-solstice activity and precipitation, predicts delays of +0.8 days per ${ }^{\circ} \mathrm{C}$, while the pre-solstice model predicts advances of -1.0 days per ${ }^{\circ} \mathrm{C}$, demonstrating that information on both pre- and post-solstice climate is necessary to reproduce the observed $\mathrm{EOS}_{50}$ responses to rising temperature.

The offsetting effect of pre-solstice development on autumn-warming-induced delays in EOS $_{50}$ also is apparent when quantifying either regional trends over the past 70 years (time series and species as random effects; Fig. S8) or spatial patterns in EOS $_{50}$ (year and species as random effects; Fig. S9). On average, European $\mathrm{EOS}_{50}$ dates have been delayed by only $+0.35 \pm 0.02$ days per decade (Fig. S8b). Yet, when keeping pre-solstice productivity constant by including it as a fixed effect in addition to year, the model predicts a delay of $+0.81 \pm 0.03$ days per decade (Fig. S8d), showing that the increase in pre-solstice vegetation activity has offset up to $\sim 60 \%$ of the delay in $\mathrm{EOS}_{50}$ that would have occurred if pre-solstice productivity had not increased. This explains why $\mathrm{EOS}_{50}$ delays have contributed only $\sim 15 \%$ (2.4 \pm 0.2 days) to the $16.7 \pm 0.4$ day-long extension of the growing season that has occurred over the past 70 years (Fig. S8a, b). The advancing effect of pre-solstice productivity on EOS dates is also evident across spatial gradients (Fig. S9 a,c), such that $\mathrm{EOS}_{50}$ dates occur only $+0.85 \pm 0.03$ days later for each $1^{\circ} \mathrm{C}$ increase in that region's autumn temperature, while they occur $+1.40 \pm 0.04$ days ${ }^{\circ} \mathrm{C}-1$ later if the advancing effect of pre-solstice productivity is removed (39\% reduction of the geographic autumn temperature response; Fig. S9 b,d).

The progression of senescence is modulated by autumn air temperature, as is evident from the delaying effect of warm autumns on $\mathrm{EOS}_{50}$ dates (Figs. 2, 3 and 5 and Extended Data Fig. 6b,c). However, if increased pre-solstice vegetation activity and development (Extended Data Fig. 5a-b) indeed is the main driver of an earlier onset of EOS, one should find an ever earlier susceptibility of trees to autumn cooling. To test this, we conducted temporal moving-window analyses on the European long-term observations showing whether the reversal date - at which increased productivity and temperature start to be associated with delayed $\mathrm{EOS}_{50}$ dates - has shifted over recent decades. The results reveal that this is the case, with reversal dates shifting to earlier dates by an average of -0.6 to -1.0 days per year (Fig. $3 f$ and Figs. S5fS7f or Fig. S10b for a shorter moving window). It is also reflected in the moving windows of monthly effect sizes, which show that July photosynthesis, temperature and radiation have been more strongly associated with delayed $\mathrm{EOS}_{50}$ dates over recent decades (Fig. 3c and Figs. S5c-S7c). As an alternative method of determining when autumn cooling starts driving senescence progression, we modelled the autumn period best explaining $\mathrm{EOS}_{50}$ dates and found that it has advanced by $-0.20 \pm 0.07$ days per year for the 19662015 period (Extended Data Fig. 7a) or by $-0.43 \pm 0.09$ days per year for the 1981-2015 period (Extended Data Fig. 7b). This earlier start of the period when trees react to autumn cooling provides further evidence for an earlier onset of senescence in response to increased early-season development.

Previous research has suggested a negative feedback between growing-season activity and autumn phenology, with increased activity driving earlier EOS 9 . These findings came from monitoring of European deciduous trees and neglected possible mediating effects of the annual day length cycle. The satellite data analysed here now reveal such negative feedback across the entire Northern Hemisphere temperate and boreal forest biome, implying a widespread constraint on future growing-season extensions in response to global warming. To disentangle the environmental drivers of this negative feedback, we ran univariate (Extended Data Fig. 8) and multiple linear regression models that included air temperature, solar radiation, and water availability in addition to spring budburst dates as predictor variables, all of which factors have been shown to affect leaf senescence dates ${ }^{8-10,21,35-37}$ (Fig. 5 and Extended Data Fig. 9 or Fig. S11 using soil moisture instead of precipitation to represent water availability). The results showed that leaf-out timing, cumulative radiation, and water availability had small effects on $\mathrm{EOS}_{10}$ and $\mathrm{EOS}_{50}$ dates (Fig. 5), suggesting that temperature-driven development and growth, rather than a constrained leaf lifespan, 
186

187

188

189

190

191

192

193

194

195

196

197

198

199

200

201

202

203

204

205

206

207

208

209

210

211

212

213

214

215

216

217

218

219

220

221

222

223

224

225

226

227

228

229

230

231

232

233

234

235

236 radiation-induced leaf ageing, or drought effects, is driving the advancing effect of early-season vegetation activity on autumn phenology.

To further isolate the mechanisms driving the discovered reversal of the effects of global warming around the June solstice, we designed two experiments using a dominant European tree (Fagus sylvatica). In a first experiment, we cooled (day- / night-time temperature $\left.=10^{\circ} \mathrm{C} / 5^{\circ} \mathrm{C}\right)$ and shaded $(\sim 84 \%$ light reduction) saplings during different times of the season. Pre-solstice temperature again had a strong advancing effect on autumn phenology, with cooling of trees in June causing a delay in $\mathrm{EOS}_{10}$ and $\mathrm{EOS}_{50}$ dates of $+16.5 \pm 6.6$ days and $+10.2 \pm 2.5$ days (mean \pm standard error), respectively, whereas cooling in July had no effect and August cooling tended to advance EOS dates (Fig. 4a and S12a), in full agreement with the global-scale remote sensing data and the European ground observations. The effect of shading was small before the June solstice and most pronounced during July - the month with the highest mean daily radiation and temperature - with $+6.5 \pm 2.8$ days later $\mathrm{EOS}_{50}$ under shade conditions. Radiation effects thus followed a different seasonal pattern than temperature, supporting a direct effect of radiation on leaf ageing 22,36 . Summer photosynthesis was equally reduced in both the shade and the temperature treatments by $52-72 \%$ compared to the control (Fig. S13). That pre-solstice temperature, but not pre-solstice light availability, affected EOS dates provides further support for the idea that accelerated growth and development under warmer temperatures, rather than photosynthesis- or UV-induced leaf ageing, are the main drivers of the pre-solstice effects on senescence dates.

In a second experiment, we tested the effects of elevated sink strength (nutrient addition) and soil moisture. In agreement with the idea that nitrogen supply governs the sink control of leaf development and senescence ${ }^{19}$, there was a strong delaying effect of extra nutrients on EOS dates, with $+22 \pm 6$-day (mean \pm standard error) later $\mathrm{EOS}_{10}$ and $+14 \pm 5$-day later $\mathrm{EOS}_{50}$ in trees grown in nutrient-rich soils compared to trees grown in NPK-poor soils (Extended Data Fig. 10 and Fig. S14). Reduced soil moisture slightly delayed EOS dates (by 2 days) up to a threshold at which complete soil dehydration led to premature leaf die-off (Extended Data Fig. 10 and Fig. S15). These experimental findings match our findings from European phenological gardens and satellite-derived in situ observations, in which direct precipitation or soil moisture had smaller effects than air temperature and early-season growth.

In conclusion, our Northern-Hemisphere-wide investigation of the seasonal drivers of autumn phenology shows a consistent reversal in the effects of global warming on leaf senescence timing after the summer solstice, present i) across large biogeographic ranges with varying pre-solstice growth (satellite data), ii) in four tree species with different spring phenologies (ground data), and iii) under controlled experimental conditions. These findings lend strong support to the idea that the annual day-length cycle mediates the seasonal control on EOS by vegetation activity and air temperature (Solstice-as-PhenologySwitch [SPS] hypothesis; Fig. 1). Hence, EOS variations emerge as the result of a complex synchronization between a trees' developmental state, seasonal variation in the circadian rhythm and climate fluctuations. This mediation provides a unifying framework to explain previous results on the seasonal effects of temperature on autumn phenology, in which the magnitude and direction of the temperature effect varied depending on the season $15,17,18,31,38,39$. Across the whole temperate and boreal forest area, warmer presolstice temperatures drive an earlier onset of senescence, while warmer post-solstice temperatures slow down the progression of senescence, suggesting that senescence will start earlier but progress more slowly in the future.

The reversal in how trees respond to temperature during the summer likely evolved as an adaptation to seasonal climates with harsh winters because it allows plants to reliably predict the progression of the seasons and prepare for winter dormancy long before the temperature actually starts dropping 29 . A response shift around the June solstice enables trees to initiate tissue maturation and the physiological processes of leaf senescence and nutrient resorption ${ }^{26}$ in a fine-tuned balance between source and sink dynamics. Trees' physiological response to the annual day length cycle essentially calibrates their seasonal rhythms and mediates how they react to warm or cool temperatures, now and in the future. Improved models of plant development and growth under climate change will need to incorporate the reversal of warming effects around the summer solstice. 
237

238

239

240

241

242

243

244

245

246

247

248

249

250

251

252

253

254

255

256

257

258

259

260

261

262

263

264

265

266

267

268

269

270

271

272

273

274

275

276

277

278

279

280

281

282

283

284

285

286

287

288

289

290

\section{Methods}

\section{Analysis of ground-sourced European phenology observations}

Phenology data. Direct observations of spring leaf-out and autumn leaf senescence dates for four dominant tree species from 1951-2015 were collected from the Pan European Phenology Project (www.pep725.eu) ${ }^{32}$, which provides open-access phenological data for Europe (mainly Germany, Switzerland, and Austria; Fig. S16). The start-of-season (SOS) was defined as the date when the first leaf stalks are visible (BBCH11). For a few time series, $\mathrm{BBCH} 11$ data was not available, and we used the date when $50 \%$ of leaf stalks are visible $(\mathrm{BBCH} 13)$ instead. The end-of-season $\left(\mathrm{EOS}_{50}\right)$ corresponded to the date when $50 \%$ of leaves had lost their green color (BBCH94). Alternatively, for time series for which BBCH94 data was not available, we used the date when $50 \%$ of leaves had fallen $(\mathrm{BBCH} 95)$.

Data cleaning. Following ref40, we removed (i) individual time series with fewer than 15 years of leaf-out and leaf senescence observations, (ii) dates deviating from an individual's median more than 4 times the median absolute deviation, and (iii) time series for which the standard deviation of phenological observations across years was higher than 20 for leaf-out and 25 for leaf senescence. The thresholds differed because the mean absolute deviation for leaf-out time series was lower than that for leaf senescence. In total, we kept records of the four most abundant tree species in the database (Aesculus hippocastanum, Betula pendula, Fagus sylvatica, and Quercus robur) at 3,851 sites across Central Europe, resulting in a total of 396,411 phenological observations (individuals $x$ year) and 12,759 individual time series (with a median length of 33 years; minimally 15 years, maximally 65 years) during the period 1951-2015 (Fig. S16). The number of time series per species was 3,477 for Aesculus hippocastanum, 3,375 for Betula pendula, 3,050 for Fagus sylvatica, and 2,857 for Quercus robur.

Climate data. For each site, information on air temperature, precipitation, air humidity, net and downward short-wave radiation, net long-wave radiation, and soil moisture (at $0-10 \mathrm{~cm}$ and $10-40 \mathrm{~cm}$ depth) at a spatial resolution of 0.25 arc degrees (approximately $25 \mathrm{~km}$ at the equator) and a cadence of 3 hours were derived from the Global Land Data Assimilation System (GLDAS) ${ }^{41}$ for the period 1951-2015. We then calculated daily means for each of the climate variables and the day-time $\left(T_{\text {day }}\right)$ or night-time $\left(T_{\text {night }}\right)$ temperature. To obtain $T_{\text {day }}$ and $T_{\text {night }}$, we approximated hourly temperatures using the "stack_hourly_temps" $R$ function from the chillR package ${ }^{42}$, and then calculated the means of all temperatures from sunrise to sunset or sunset to sunrise, respectively, whereby sunset and sunrise were calculated with the "daylength" function (chillR package) based on latitude and day-of-year information. Means of monthly atmospheric $\mathrm{CO}_{2}$ concentrations for each degree of latitude were taken from the CMIP6 concentration dataset ${ }^{43}$. To represent soil water availability in the photosynthesis model (see next section), for each site, we extracted physical soil properties (clay and coarse fragment content) from world maps at $250 \mathrm{~m} \mathrm{spatial}$ resolution through SoilGrids ${ }^{44}$. Soil texture was computed based on the texture-to-parameter conversion by ref ${ }^{45}$, using the Database of Hydraulic Properties of European Soils (HYPRES) implemented in the R package soiltexture ${ }^{46}$.

Photosynthesis calculation. We derived daily net daytime photosynthesis estimates (excluding night-time respiration) [Anet day] from two photosynthesis models, hereafter referred to as LPJ model and P model.

LPJ model: Anet day was computed using empirical information on $\mathrm{T}_{\text {day }}$, minimum and maximum daily temperature, air humidity, atmospheric $\mathrm{CO}_{2}$, short- and long-wave radiation, and soil moisture, following the coupled photosynthesis and stomatal conductance sub-model of LPJ-GUESS ${ }^{47}$. The temperature response of photosynthesis followed ref ${ }^{48}$, with the minimum temperature limit at $1^{\circ} \mathrm{C}$, the maximum at $45^{\circ} \mathrm{C}$, and the temperature optimum between $18^{\circ} \mathrm{C}$ and $25^{\circ} \mathrm{C}$ (see Fig. S17). To account for the effect of water availability on photosynthesis ${ }^{49}$, we included a daily water stress factor in our seasonal photosynthesis estimates, which was calculated following the water balance module of LPJ-GUESS50. The fraction of absorbed photosynthetically active radiation (fAPAR) was set to 0 before SOS and to 0.5 after the SOS date, since nearly half of the short-wave radiation is intercepted by the canopy ${ }^{45}$. See Supplementary Materials in Zani et al. ${ }^{9}$ for a detailed description of the model and Github for the annotated R code.

$P$ model (developed by Stocker et al. ${ }^{34}$ and following the theory of refs 51,52$)$ : Photosynthesis was computed as a function of $\mathrm{T}_{\text {day }}$, vapor pressure deficit, atmospheric $\mathrm{CO}_{2}$, short-wave radiation, and relative soil 
291

292

293

294

295

296

297

298

299

300

301

302

303

304

305

306

307

308

309

310

311

312

313

314

315

316

317

318

319

320

321

322

323

324

325

326

327

328

329

330

331

332

333

334

335

336

337

338

339

340

341

342

343

344

moisture (actual moisture relative to field capacity). In addition, information on elevation and the site-level ratio of actual over potential annual mean evapotranspiration was included to represent atmospheric pressure and average local aridity, respectively. Apparent quantum yield efficiency was set to 0.087 , optimal leaf-internal/ambient $\mathrm{CO}_{2}$ ratios (ci/ca) were calculated based on the method developed in ref51, and limitations in the photosynthetic capacity for electron transport (Jmax) were accounted for based on the method developed in ref52. As for the LPJ model, fAPAR was set to 0 before SOS. The P model was run via the rpmodel function in the $R$ package rpmodel 34 .

Analysis. In total, we included seven variables in our analyses: LPJ-model photosynthesis, P-model photosynthesis, $\mathrm{T}_{\text {day }}$ and $\mathrm{T}_{\text {night }}$, short-wave radiation, $\mathrm{CO}_{2}$ levels, and precipitation.

Seasonal and monthly photosynthesis/climate: To obtain monthly photosynthesis, $T_{\text {day }}$, and short-wave radiation values, we took the sums of daily photosynthesis and radiation values and the means of daily $T_{\text {day }}$ values for each month (January to October) [see e.g., Fig. 3a, b]. Similarly, we aggregated values of these variables for six 30-day intervals before, during, and after the June solstice (with 10-day steps), i.e., from May 13 to June 11, May 23 to June 21, June 2 to July 1, June 12 to July 11, June 22 to July 21, and July 2 to July 31 (see e.g., Fig. 3d). In addition, we summed the daily photosynthesis values for eight periods (SOS to May 22, SOS to June 21 [solstice], SOS to July 21, SOS to August 20, SOS to mean EOS 50 , May 22 to mean $\mathrm{EOS}_{50}$, June 21 [solstice] to mean $\mathrm{EOS}_{50}$, and July 21 to mean $\mathrm{EOS}_{50}$ ) [see e.g., Fig. 3e]. For $\mathrm{T}_{\text {day }}$ and short-wave radiation we calculated the means for eight periods starting on 21 March instead of the variable SOS date.

Autumn temperature intervals: It is well known that cold temperatures at the end of the season accelerate the senescence process ${ }^{9,17,18}$, and, for each time series, we therefore determined the optimal autumn interval for which temperature explains most of the variation in $\mathrm{EOS}_{50}$ dates. To do so, we ran linear regressions between $\mathrm{EOS}_{50}$ dates and the temperature interval ( $T_{\text {day }}$ or $\left.T_{\text {night }}\right) 10$ to 120 days before the average $\mathrm{EOS}_{50}$ date of each time series (with 10-day steps). Relationships were evaluated using both the coefficient of determination $\left(R^{2}\right)$ values and the standardized coefficients (Extended Data Fig. 6c). These analyses showed slightly higher $R^{2} s$ and standardized coefficients for $T_{\text {night }}$ than for $T_{\text {day }}$, and, for each time series, we therefore included the respective $T_{\text {night }}$ interval with the highest $R^{2}$ in the analyses (hereafter referred to as Autumn $T_{\text {night, }}$, see e.g., Fig. 3h).

Models: To test for the effects of photosynthesis and climate parameters during different times of the year on $\mathrm{EOS}_{50}$ dates, we ran linear mixed models, implemented in the R package Ime453. All models included either both time series and species random effects, or only a time series random effect in case the model was run separately for each species. Both predictor and dependent variables were standardized - by subtracting the mean and dividing by the standard deviation - to obtain relative effect sizes. For the monthly models of photosynthesis, March to October values were included as predictors in the model, with March values representing the sum of January to March (Figs. 3 and S5). This aggregation was done because no or only very little photosynthesis occurred during the January-to-March period (average leaf-out date across all observations: 26 April). For the monthly $T_{\text {day }}$ model, January to October values were included as predictors (Fig. S6a,b). To characterize the effects of photosynthesis, $T_{\text {day }}$, and short-wave radiation within 30-day-long intervals around the June solstice, we included the variable value within the respective interval and Autumn $\mathrm{T}_{\text {night }}$ (to control for autumn temperature) as fixed effects (Figs. 3d and S5d-S7d). To characterize the seasonal effects of photosynthesis, $T_{\text {day }}$, and short-wave radiation, we included the variable value within the respective interval as single fixed effect (Figs. 3e and S5e-S7e).

Finally, we ran mixed models based on the seasonal periods that emerged as the strongest drivers of leaf senescence, i.e., pre-solstice (leaf-out or March 21 to June 21) and post-solstice climate (June 22 to mean senescence date). Models were run separately for photosynthesis, $T_{\text {day }}$ and short-wave radiation, and we accounted for possible effects of water availability, atmospheric $\mathrm{CO}_{2}$ concentration, and autumn temperature by additionally including the sums of pre-solstice (March 20 to June 21) and post-solstice (June 21 to mean time series senescence date) precipitation, annual $\mathrm{CO}_{2}$ concentration, and Autumn $\mathrm{T}_{\text {night }}$ as fixed effects (full model; Fig. $3 \mathrm{~g}$ ). To test for the individual effects of pre-solstice and post-solstice variables on model performance and predictions, we additionally ran models that only included either pre-solstice 
photosynthesis, $\mathrm{T}_{\text {day }}$, or radiation and pre-solstice precipitation (pre-solstice model) or post-solstice photosynthesis, $\mathrm{T}_{\text {day }}$, or radiation and post-solstice precipitation (post-solstice model; Figs. $3 \mathrm{~g}$ and S5g$\mathrm{S} 7 \mathrm{~g}$ ). To test for multicollinearity among covariates, we calculated variance-inflation factors - using the vif function implemented in the $\mathrm{R}$ package $\mathrm{car}^{54}$, for all models that contained multiple variables, i.e., the monthly and the full models. All VIFs were $<2$, indicating sufficient independence among predictors.

Moving-window analysis: To test whether the relative effects of variables have been changing over the past decades, we additionally ran all above-mentioned mixed models separately for each 20-year time period from 1966-2015. To ensure that the long-term average climate of sites did not differ across the movingwindow periods as a result of site-level differences, we excluded high-elevation sites $>600 \mathrm{~m}$ a.s.l. as they were underrepresented in earlier years. We then tested whether the average long-term (1948-2015) climate of sites included in each moving-window period differed between years and found no trend (Fig. S18), demonstrating that that there is no systematic bias in the average site-level climate conditions among moving windows. Within each 20-year moving-window, time series with fewer than 15 years of observations were excluded. To ensure that all sites were equally represented in each moving-window period, we ran a reduced analysis with 15-year moving-window intervals for the 1980-2015 period, including only time series for which at least 30 years of observations were available in total during 1980-2015 and for which at least 12 years were available during each 15-year moving window (Fig. S10).

To estimate the reversal date of the climate-autumn phenology relationship at which increased temperature and productivity start to be associated with delayed $\mathrm{EOS}_{50}$ dates, we conducted movingwindow analyses of the monthly photosynthesis/climate effects. To do so, for each 20-year (Fig. 3f) or 15year (Fig. S10b) moving-window, we interpolated the date at which the effect of monthly photosynthesis/climate crosses the zero line during summer (see e.g., Fig. 3a). The dates were inferred both across species (based on models including time series and species random effects) and separately for each species (based on models including only time series random effects; see e.g., Fig. 3f). To estimate the overall trend in the reversal dates over time, we ran a linear regression between the reversal date obtained for each moving window and year.

As an alternative to estimating the day at which autumn temperature starts driving senescence progression, for each moving window, we estimated the autumn temperature-sensitive period, based on the autumn period for which temperature best explained variation in $\mathrm{EOS}_{50}$ dates (see above paragraph on Autumn temperature intervals) [Extended Data Fig. 7].

\section{Analysis of satellite-derived phenology observations}

Phenology data. Spring leaf-out and autumn leaf senescence dates for the period 2001-2018 were collected from the MODIS Global Vegetation Phenology product (MCD12Q2 V6) ${ }^{55}$, which provides phenological metrics at $500 \mathrm{~m}$ spatial resolution for every vegetated pixel on land derived from time series of the 2-band Enhanced Vegetation Index (EVI2) calculated from MODIS Nadir Bidirectional Reflectance Distribution Function (BRDF)-Adjusted Reflectance (NBAR). SOS was defined as the date when EVI2 first crossed $15 \%$ of the segment EVI2 amplitude greenness. EOS was defined as the date when EVI2 last dropped by $10 \%\left(E O S_{10}\right)$ or $50 \%\left(E O S_{50}\right)$ of the segment $E V I 2$ amplitude, representing the start of senescence and mid-greendown, respectively.

Data cleaning. We extracted phenology information for all Northern Hemisphere pixels classified as mixed, broadleaf deciduous, evergreen needleleaf, or deciduous needleleaf forest (tree cover $>60 \%$ ) by the MODIS land cover type map (MCD12Q1 V6) ${ }^{56}$. We removed pixels (i) for which phenology information for fewer than 15 years was available and (ii) for which the mean SOS occurred before March 1 or after May 31 and for which the mean EOS ${ }_{50}$ occurred before July 18 and after November 30 . We then aggregated the data to 0.25 arc-degree $(27.8 \mathrm{~km}$ at the Equator) spatial resolution to match with the resolution of the climate data. This resulted in a total of 15,459 pixels of which $60 \%(9,240)$ are classified as mixed forest, $19 \%$ $(2,958)$ as broadleaf deciduous forest, $19 \%(3,004)$ as evergreen needleleaf forest, and $2 \%(257)$ as deciduous needleleaf forest. 
Analysis. As for the ground-sourced phenology data, climate and soil moisture information at a spatial resolution of 0.25 arc degrees were derived from the Global Land Data Assimilation System (GLDAS) ${ }^{41}$. Information on gross primary production (GPP) was extracted from the MODIS Gross Primary Productivity product (MOD17A2H V6) ${ }^{33}$, which provides an 8-day composite dataset at $500 \mathrm{~m}$ spatial resolution. In total, we included eight variables in our analyses: GPP, LPJ model-derived Anet day, $T_{\text {day }}$ and $T_{\text {night, }}$, short-wave radiation, $\mathrm{CO}_{2}$ levels, precipitation, and soil moisture (at $0-40 \mathrm{~cm}$ depth).

Seasonal and monthly photosynthesis/climate: To obtain monthly photosynthesis, $\mathrm{T}_{\text {day }}$, and short-wave radiation values, we took the sums (GPP, LPJ model Anet $t_{\text {day }}$, and $P$ model Anet $_{\text {day }}$ ) or means ( $T_{\text {day }}$, shortwave radiation) of daily values for each month. Similarly, we aggregated values of these variables for six 30-day intervals with 10-day moving steps around the June solstice (see 1.5.1 for details).

Autumn temperature intervals: The optimal autumn intervals at which temperature has the strongest effect on senescence dates were calculated as described in the above section Analysis of ground-sourced European phenology observations - Autumn temperature intervals. For $\mathrm{EOS}_{50}$ dates, these analyses showed slightly higher $\mathrm{R}^{2} \mathrm{~s}$ and standardized coefficients for $\mathrm{T}_{\text {day }}$ than for $\mathrm{T}_{\text {night }}$ (Extended Data Fig. 6b), and, for each time series, we therefore included the respective $T_{\text {day }}$ interval with the highest $R^{2}$ in the analyses (hereafter referred to as Autumn $\mathrm{T}_{\text {day }}$, Extended Data Fig. 2h,j and Figs. S2h and S4h). For EOS ${ }_{10}$ dates, we found no delaying effect of any autumn temperature interval (Extended Data Fig. 6a) and we therefore did not control for autumn temperature in the analyses.

Models: To test for the effects of photosynthesis and climate parameters during different times of the year on autumn senescence dates, we ran pixel-level linear models. Both predictor and dependent variables were standardized to obtain relative effect sizes. To run the monthly models, we included the relative photosynthesis/climate value of each month (January to October) in a multivariate model. For the monthly effects of photosynthesis and short-wave radiation on $\mathrm{EOS}_{10}$ dates, only April to September values were included as predictors in the model, with April values representing the sums of January to April (Fig. $2 \mathrm{~g}$ ). To characterize the effects of photosynthesis, $T_{\text {day }}$, and short-wave radiation within 30-day-long intervals around the June solstice on $\mathrm{EOS}_{10}$ dates, we included the variable value within the respective interval as single fixed effect (see e.g., Fig. $2 \mathrm{~h}$ ), whereas we additionally included Autumn $\mathrm{T}_{\text {day }}$ (to control for autumn temperature) as fixed effect when testing for the effects on EOS ${ }_{50}$ dates (see e.g., Extended Data Fig. 2). We then ran models including information on pre-solstice (sum of photosynthesis from leaf-out to June 21 or mean of $\mathrm{T}_{\text {day }}$ and radiation from March 20 to June 21) and post-solstice vegetation activity / climate (sum or mean from June 22 to mean senescence date). Models were run separately for photosynthesis, $T_{\text {day }}$ and short-wave radiation, and we accounted for possible effects of water availability, atmospheric $\mathrm{CO}_{2}$ concentration, and autumn temperature by additionally including the sums of pre-solstice (March 20 to June 21) and post-solstice (June 22 to mean time series senescence date) precipitation, annual $\mathrm{CO}_{2}$ concentration, and Autumn $\mathrm{T}_{\text {day }}$ (only for $\mathrm{EOS}_{50}$ dates) as fixed effects (full model; Fig. 2f). To test for the explanatory power of pre-solstice variables on inter-annual variation in the timing of EOS, we ran univariate models that included either pre-solstice GPP, $T_{\text {day }}$, radiation, soil moisture or leaf-out date as single effect (Extended Dta Fig. 8).

To approximate the end of the period during which early-season vegetation activity exhibited an advancing effect on the onset of senescence, we ran pixel-level linear regression models between gross primary productivity (GPP) and EOS ${ }_{10}$ dates. GPP summation always started the day of leaf-out and ended at different dates, using 30-day steps. For each pixel, we then kept the period with the most negative (advancing) effect on $\mathrm{EOS}_{10}$ dates. Extended Data Fig. 4 shows the estimated end date (red line) and starting date (leaf-out date; green line) of this period along latitude, whereby pixel-level estimates were averaged for each degree latitude.

To test for decadal-scale temporal trends in EOS dates, we additionally ran mixed effects models where pixels are treated as grouping variables of random intercepts, including only year or year and presolstice photosynthesis, temperature or radiation as fixed effects (Fig. 2i-k). 
452

453

454

455

456

457

458

459

460

461

462

463

464

465

466

467

468

469

470

471

472

473

474

475

476

477

478

479

480

481

482

483

484

485

486

487

488

489

490

491

492

493

494

495

496

497

498

499

500

501

502

503

504

\section{Experiments}

Experiment 1 - Seasonal temperature and light manipulation. The experiment was conducted in Zurich, Switzerland, between March and December 2021. Three-year old Fagus sylvatica trees were used to observe the seasonal effects of cooling and shading ( 9 treatments: 1 control, 4 temperature and 4 shade treatments) on autumn phenology. Plants were obtained from a local nursery in February 2021, transferred to 20 I plastic pots containing a 1:1:1 sand / peat / organic soil mixture with a Nitrogen $(\mathrm{N})$ concentration of $\sim 65 \mathrm{~g} \mathrm{~m}^{-3}$, a Phosphate $\left(\mathrm{P}_{2} \mathrm{O}_{5}\right)$ concentration of $\sim 140 \mathrm{~g} \mathrm{~m}^{-3}$, and a Potassium $\left(\mathrm{K}_{2} \mathrm{O}\right)$ concentration of $\sim 400$ $\mathrm{g} \mathrm{m}^{-3}$.

We exposed trees to cooling or shading conditions during four 1 month-long periods (30 April - 30 May [hereafter referred to as May treatment], 26 May - 25 June [June treatment], 26 June - 26 July [July treatment], and 27 July -26 August [August treatment]). The experimental and observational unit was a pot with a single individual. The control consisted of 38 trees growing outside under full sun conditions. The treatments consisted of 14 trees each. In the cooling treatments, trees were kept in a climate chamber with a night-time temperature of $5^{\circ} \mathrm{C}$ and a day-time temperature of $10^{\circ} \mathrm{C}$, and simulating ambient day length and light intensities. In the shading treatments, trees were exposed to shade conditions using a shading net that intercepted c. $84 \%( \pm 10 \%$; mean \pm SD) of the PAR experienced by the control treatment. All trees were watered regularly to keep soil moisture constant.

To quantify seasonal changes in leaf chlorophyll content, we measured the relative chlorophyll content at, on average, $4 \mathrm{wk}$ intervals during summer and $1 \mathrm{wk}$ intervals from mid-September until December with a SPAD-502 Plus (Soil Plant Analysis Development, Minolta Camera Co., Ltd, Tokyo, Japan). Per individual, SPAD readings from nine leaves were averaged, whereby we randomly selected three leaves from the top, middle and lower parts of the tree crown. SPAD readings were then transferred to total chlorophyll content ( $\mathrm{Ch} / \mathrm{in} \mu \mathrm{g} / \mathrm{g}$ fresh weight) using an empirically established equation for Fagus sylvatica leaves ${ }^{57}$ :

$$
\text { Chl }=-0.0029 \times S P A D^{2}+1.175 \times S P A D+3.8506
$$

To calculate individual-level leaf chlorophyll content, we additionally included the percentage of leaf abscission in autumn for each individual, that is, the already-dropped leaves in autumn have a chlorophyll content of 0 . For example, an individual that lost already $50 \%$ of leaves with a chlorophyll content of 30 for the remaining leaves has a total chlorophyll content of $15(30 \times 0.5)$. The chlorophyll content between two consecutive measurement dates was estimated using linear interpolation. Finally, we calculated individual leaf senescence dates as the day-of-year when chlorophyll content last fell below $90 \%\left(E^{2} S_{10}\right)$ or $50 \%$ $\left(E O S_{50}\right)$ of the maximum chlorophyll content in summer. Because the shading treatment caused short-term changes in chlorophyll content that do not reflect autumn phenological responses, chlorophyll measurements during the treatment until two weeks after the respective treatment period were excluded for the determination of autumn phenology.

Leaf net photosynthesis $\left(\mathrm{CO}_{2}\right.$ uptake per leaf area) was monitored on a monthly basis from May to July on one leaf of 3-13 individuals per treatment using a portable infrared gas analyzer (LI-6800, Li- Cor, Lincoln, NE) with a $3 \times 3 \mathrm{~cm}$ leaf chamber. Leaf chamber temperature during the measurements reflected the mean day-time $(8.00-15.00)$ temperature experienced by each treatment in the respective month, leaf chamber light intensities reflected the mean light intensities experienced by each treatment in the respective month. Humidity was set to $65 \%$, leaf chamber CO2 to $400 \mathrm{ppm}$. Measurements were taken between 2 to 6 hours after sunrise with replicate individuals of each treatment done on four consecutive days.

To test for differences in leaf senescence dates among treatments, we ran multivariate linear models including temperature and shade treatments as categorical variables.

Experiment 2 - Nutrient and soil moisture manipulation. The experiment was conducted in Zurich, Switzerland (450 $\mathrm{m}$ a.s.I.) between June and December 2020. Sixty three-year old Fagus sylvatica trees were obtained from a local nursery in February 2020, transferred to 20 I plastic pots containing a 50/50 sand/peat mixture, and kept outdoors under uniform conditions until the start of the experiment (15 June 2020). Due to Covid19 restrictions, the experiment could not begin until June 15, but all trees were watered once a week to keep soil moisture constant. During the experiment (June 15 - December 1), all trees were kept outdoors and arranged in randomized blocks. 
The experiment followed a $2 \times 4$ full-factorial design with two nutrient treatments (high versus low nitrogen, phosphorous, and potassium) and four irrigation treatments (High, Intermediate, Low, and No irrigation), resulting in a total of eight treatment combinations (Extended Data Fig. 10). The experimental and observational unit was a pot with a single individual. Each irrigation treatment consisted of 14 individuals of which eight individuals were exposed to high-nutrient and seven to low-nutrient conditions. In the High Irrigation treatment, plants were watered regularly (on average, every 3rd day); in the Intermediate Irrigation treatment, plants were watered every other time (i.e., every 6 days); in the Low Irrigation treatment, plants were watered every fourth time (i.e., every $\sim 12$ days); in the No Irrigation treatment, plants were not watered and received only natural precipitation. On June 15 and August 12, the trees of the high-nutrient treatment were fertilized with $20 \mathrm{~g}$ organic NPK fertilizer (DCM ECO-XTRA 1), containing 8\% nitrogen (1.6 g N), $5 \%$ phosphorous ( $1 \mathrm{~g} \mathrm{P}_{2} \mathrm{O}_{5}$ ) and $6 \%$ potassium $\left(1.2 \mathrm{~g} \mathrm{~K}_{2} \mathrm{O}\right)$. On July 15, all trees received $0.4 \mathrm{~g}$ micronutrients (DCM MICRO-MIX). For each irrigation treatment, soil moisture was measured weekly in 6 randomly chosen individuals. We observed strong differences in soil moisture among the irrigation treatments, with a median soil moisture between June and September of $22.1 \%, 11.4 \%, 7.6 \%$. and $4.6 \%$ for the High, Intermediate, Low, and No irrigation treatments, respectively (Fig. S15a,b).

Leaf chlorophyll content measurements and autumn phenology scoring were done as in Experiment 1. Chlorophyll measurements were conducted at, on average, 2 wk intervals during summer and 1 wk intervals during October.

To test for differences in leaf senescence dates among treatments, we ran multivariate linear models including nutrient and irrigation treatment as categorical variables. We additionally included an interaction term between treatments to test if nutrient or irrigation effects depended on the other treatment but found no significant effect $(P=0.74)$.

\section{References}

1. Menzel, A. \& Fabian, P. Growing season extended in Europe. Nature 397, 659 (1999).

2. Zohner, C. M., Mo, L., Pugh, T. A. M., Bastin, J. F. \& Crowther, T. W. Interactive climate factors restrict future increases in spring productivity of temperate and boreal trees. Glob. Chang. Biol. 26, 4042-4055 (2020).

3. Chuine, I. Why does phenology drive species distribution? Philos. Trans. R. Soc. B Biol. Sci. 365, 3149-3160 (2010).

4. Renner, S. S. \& Zohner, C. M. Climate Change and Phenological Mismatch in Trophic Interactions Among Plants, Insects, and Vertebrates. Annu. Rev. Ecol. Evol. Syst. 49, 165-182 (2018).

5. Richardson, A. D. et al. Climate change, phenology, and phenological control of vegetation feedbacks to the climate system. Agric. For. Meteorol. 169, 156-173 (2013).

6. Keenan, T. F. et al. Net carbon uptake has increased through warming-induced changes in temperate forest phenology. Nat. Clim. Chang. 4, 598-604 (2014).

7. Gallinat, A. S., Primack, R. B. \& Wagner, D. L. Autumn, the neglected season in climate change research. Trends Ecol. Evol. 30, 169-176 (2015).

8. Keenan, T. F. \& Richardson, A. D. The timing of autumn senescence is affected by the timing of spring phenology: Implications for predictive models. Glob. Chang. Biol. 21, 2634-2641 (2015).

9. Zani, D., Crowther, T. W., Mo, L., Renner, S. S. \& Zohner, C. M. Increased growing-season productivity drives earlier autumn leaf senescence in temperate trees. Science (80-. ). 370, 10661071 (2020).

10. Fu, Y. S. H. et al. Variation in leaf flushing date influences autumnal senescence and next year's flushing date in two temperate tree species. Proc. Natl. Acad. Sci. U. S. A. 111, 7355-7360 (2014).

11. Chuine, I. Why does phenology drive species distribution? Philos. Trans. R. Soc. B Biol. Sci. 365, 3149-3160 (2010).

12. Vitasse, Y. et al. The great acceleration of plant phenological shifts. Nat. Clim. Chang. (2022).

13. Delpierre, N. et al. Modelling interannual and spatial variability of leaf senescence for three deciduous tree species in France. Agric. For. Meteorol. 149, 938-948 (2009).

14. Chen, L. et al. Leaf senescence exhibits stronger climatic responses during warm than during cold autumns. Nat. Clim. Chang. (2020) doi:10.1038/s41558-020-0820-2. 
611

612

15. Liu, G., Chen, X., Zhang, Q., Lang, W. \& Delpierre, N. Antagonistic effects of growing season and autumn temperatures on the timing of leaf coloration in winter deciduous trees. Glob. Chang. Biol. 24, 3537-3545 (2018).

16. Zohner, C. M., Rockinger, A. \& Renner, S. S. Increased autumn productivity permits temperate trees to compensate for spring frost damage. New Phytol. 221, 789-795 (2019).

17. $\mathrm{Fu}, \mathrm{Y}$. H. et al. Larger temperature response of autumn leaf senescence than spring leaf-out phenology. Glob. Chang. Biol. 24, 2159-2168 (2018).

18. Zohner, C. M. \& Renner, S. S. Ongoing seasonally uneven climate warming leads to earlier autumn growth cessation in deciduous trees. Oecologia 189, 549-561 (2019).

19. Paul, M. J. \& Foyer, C. H. Sink regulation of photosynthesis. J. Exp. Bot. 52, 1383-1400 (2001).

20. Buermann, W. et al. Widespread seasonal compensation effects of spring warming on northern plant productivity. Nature 562, 110-114 (2018).

21. Bigler, C. \& Vitasse, Y. Premature leaf discoloration of European deciduous trees is caused by drought and heat in late spring and cold spells in early fall. Agric. For. Meteorol. 307, 108492 (2021).

22. Pyung, O. L., Hyo, J. K. \& Hong, G. N. Leaf senescence. Annual Review of Plant Biology vol. 58 115-136 (2007).

23. Heide, O. M. Temperature rather than photoperiod controls growth cessation and dormancy in Sorbus species. J. Exp. Bot. 62, 5397-5404 (2011).

24. Fracheboud, Y. et al. The Control of Autumn Senescence in European Aspen. Plant Physiol. 149, 1982-1991 (2009).

25. Rossi, S. et al. Conifers in cold environments synchronize maximum growth rate of tree-ring formation with day length. New Phytol. 170, 301-310 (2006).

26. Bauerle, W. L. et al. Photoperiodic regulation of the seasonal pattern of photosynthetic capacity and the implications for carbon cycling. Proc. Natl. Acad. Sci. 109, 8612-8617 (2012).

27. Etzold, S. et al. Number of growth days and not length of the growth period determines radial stem growth of temperate trees. Ecol. Lett. 00, 1-13 (2021).

28. Luo, T. et al. Summer solstice marks a seasonal shift in temperature sensitivity of stem growth and nitrogen-use efficiency in cold-limited forests. Agric. For. Meteorol. 248, 469-478 (2018).

29. Körner, C. et al. Where, why and how? Explaining the low-temperature range limits of temperate tree species. J. Ecol. 104, 1076-1088 (2016).

30. Goulden, M. L., Munger, J. W., Fan, S. M., Daube, B. C. \& Wofsy, S. C. Exchange of carbon dioxide by a deciduous forest: Response to interannual climate variability. Science (80-. ). 271, 1576-1578 (1996).

31. Zohner, C. M., Renner, S. S., Sebald, V. \& Crowther, T. W. How changes in spring and autumn phenology translate into experimental evidence of asymmetric effects. J. Ecol. 2717-2728 (2021) doi:10.1111/1365-2745.13682.

32. Templ, B. et al. Pan European Phenological database (PEP725): a single point of access for European data. Int. J. Biometeorol. 62, 1109-1113 (2018).

33. Running, S., Mu, Q. \& Zhao, M. MOD17A2H MODIS/Terra Gross Primary Productivity 8-Day L4 Global 500m SIN Grid V006 [Data set]. NASA EOSDIS Land Processes DAAC https://doi.org/10.5067/MODIS/MOD17A2H.006 (2015).

34. Stocker, B. D. et al. P-model v1.0: An optimality-based light use efficiency model for simulating ecosystem gross primary production. Geosci. Model Dev. 13, 1545-1581 (2020).

35. Wu, Z. et al. Atmospheric brightening counteracts warming-induced delays in autumn phenology of temperate trees in Europe. Glob. Ecol. Biogeogr. 30, 2477-2487 (2021).

36. Vitasse, Y. et al. Impact of microclimatic conditions and resource availability on spring and autumn phenology of temperate tree seedlings. New Phytol. 232, 537-550 (2021).

37. Massonnet, C. et al. Leafy season length is reduced by a prolonged soil water deficit but not by repeated defoliation in beech trees (Fagus sylvatica L.): comparison of response among regional populations grown in a common garden. Agric. For. Meteorol. 297, (2021).

38. Liu, Q. et al. Delayed autumn phenology in the Northern Hemisphere is related to change in both climate and spring phenology. Glob. Chang. Biol. 22, 3702-3711 (2016).

39. Liu, G., Chen, X., Fu, Y. \& Delpierre, N. Modelling leaf coloration dates over temperate China by 
613

614

615

616

617

618

619

620

621

622

623

624

625

626

627

628

629

630

631

632

633

634

635

636

637

638

639

640

641

642

643

644

645

646

647

648

649

650

651

652

653

654

655

656

657

658

659

660

661

662

663

664

665

considering effects of leafy season climate. Ecol. Modell. 394, 34-43 (2019).

40. Vitasse, Y., Signarbieux, C. \& Fu, Y. H. Global warming leads to more uniform spring phenology across elevations. Proc. Natl. Acad. Sci. 201717342 (2017) doi:10.1073/pnas.1717342115.

41. Rodell, M. et al. The Global Land Data Assimilation System. Bull. Am. Meteorol. Soc. 85, 381-394 (2004).

42. Luedeling, E. chillR: Statistical Methods for Phenology Analysis in Temperate Fruit Trees. (2021).

43. Meinshausen, M. et al. Historical greenhouse gas concentrations for climate modelling (CMIP6). Geosci. Model Dev. 10, 2057-2116 (2017).

44. Hengl, T. et al. SoilGrids250m: Global gridded soil information based on machine learning. PLoS One 12, e0169748 (2017).

45. Sitch, S. et al. Evaluation of ecosystem dynamics, plant geography and terrestrial carbon cycling in the LPJ dynamic global vegetation model. Glob. Chang. Biol. 9, 161-185 (2003).

46. Moeys, J. soiltexture: Functions for Soil Texture Plot, Classification and Transformation. (2018).

47. Smith, B. et al. Implications of incorporating $\mathrm{N}$ cycling and $\mathrm{N}$ limitations on primary production in an individual-based dynamic vegetation model. Biogeosciences 11, 2027-2054 (2014).

48. Huang, M. et al. Air temperature optima of vegetation productivity across global biomes. Nat. Ecol. Evol. 3, 772-779 (2019).

49. Xie, Y., Wang, X., Wilson, A. M. \& Silander, J. A. Predicting autumn phenology: How deciduous tree species respond to weather stressors. Agric. For. Meteorol. 250-251, 127-137 (2018).

50. Gerten, D., Schaphoff, S., Haberlandt, U., Lucht, W. \& Sitch, S. Terrestrial vegetation and water balance-hydrological evaluation of a dynamic global vegetation model. J. Hydrol. 286, 249-270 (2004).

51. Prentice, I. C., Dong, N., Gleason, S. M., Maire, V. \& Wright, I. J. Balancing the costs of carbon gain and water transport: Testing a new theoretical framework for plant functional ecology. Ecol. Lett. 17, 82-91 (2014).

52. Wang, H. et al. Towards a universal model for carbon dioxide uptake by plants. Nat. Plants 3, 734741 (2017).

53. Bates, D., Mächler, M., Bolker, B. \& Walker, S. Fitting linear mixed-effects models using Ime4. J. Stat. Softw. 67, 1-48 (2015).

54. Mann, H. B. Nonparametric tests against trend. Econometrica 13, 245-259 (1945).

55. Friedl, M., Gray, J. \& Sulla-Menashe, D. MCD12Q2 MODIS/Terra+Aqua Land Cover Dynamics Yearly L3 Global 500m SIN Grid V006. distributed by NASA EOSDIS Land Processes DAAC https://doi.org/10.5067/MODIS/MCD12Q2.006 (2019).

56. Friedl, M. \& Sulla-Menashe, D. MCD12Q1 MODIS/Terra+Aqua Land Cover Type Yearly L3 Global $500 \mathrm{~m}$ SIN Grid V006. distributed by NASA EOSDIS Land Processes DAAC https://doi.org/10.5067/MODIS/MCD12Q1.006 (2019).

57. Percival, G. C., Keary, I. P. \& Noviss, K. The potential of a chlorophyll content SPAD meter to quantify nutrient stress in foliar tissue of sycamore (Acer pseudoplatanus), English oak (Quercus robur), and European beech (Fagus sylvatica). Arboric. Urban For. 34, 89-100 (2008).

\section{Acknowledgements}

CMZ was funded by the Ambizione grant PZ00P3_193646. TWC was funded by DOB Ecology. DP was supported by the Faculty of Science Foundation of the Charles University and the Hlávka Foundation. YHF was supported by the National Science Fund for Distinguished Young Scholars (42025101). We thank Christian Körner and V. Sebald for comments on the manuscript.

Statement of authorship: CMZ conceived and developed the study and wrote the first draft of the manuscript. LM contributed to the development of the study and the remote-sensing analysis. RB, DP and $\mathrm{CMZ}$ conducted the experiments. LMo contributed to the analysis. TWC, SSR, LM, YV, YHF and BDS contributed to the writing. 
666 Data and materials availability: All source code and experimental data are available on github

667 (https://github.com/ConstantinZohner/AutumnPhenologySPS). The PEP725 data used for this study are 668 freely available at www.PEP725.eu. The satellite-derived phenology observations are freely available at 669 https://doi.org/10.5067/MODIS/MCD12Q2.006.

670

671 Competing interests: The authors declare that there are no competing interests.

672 
673

674

675

676

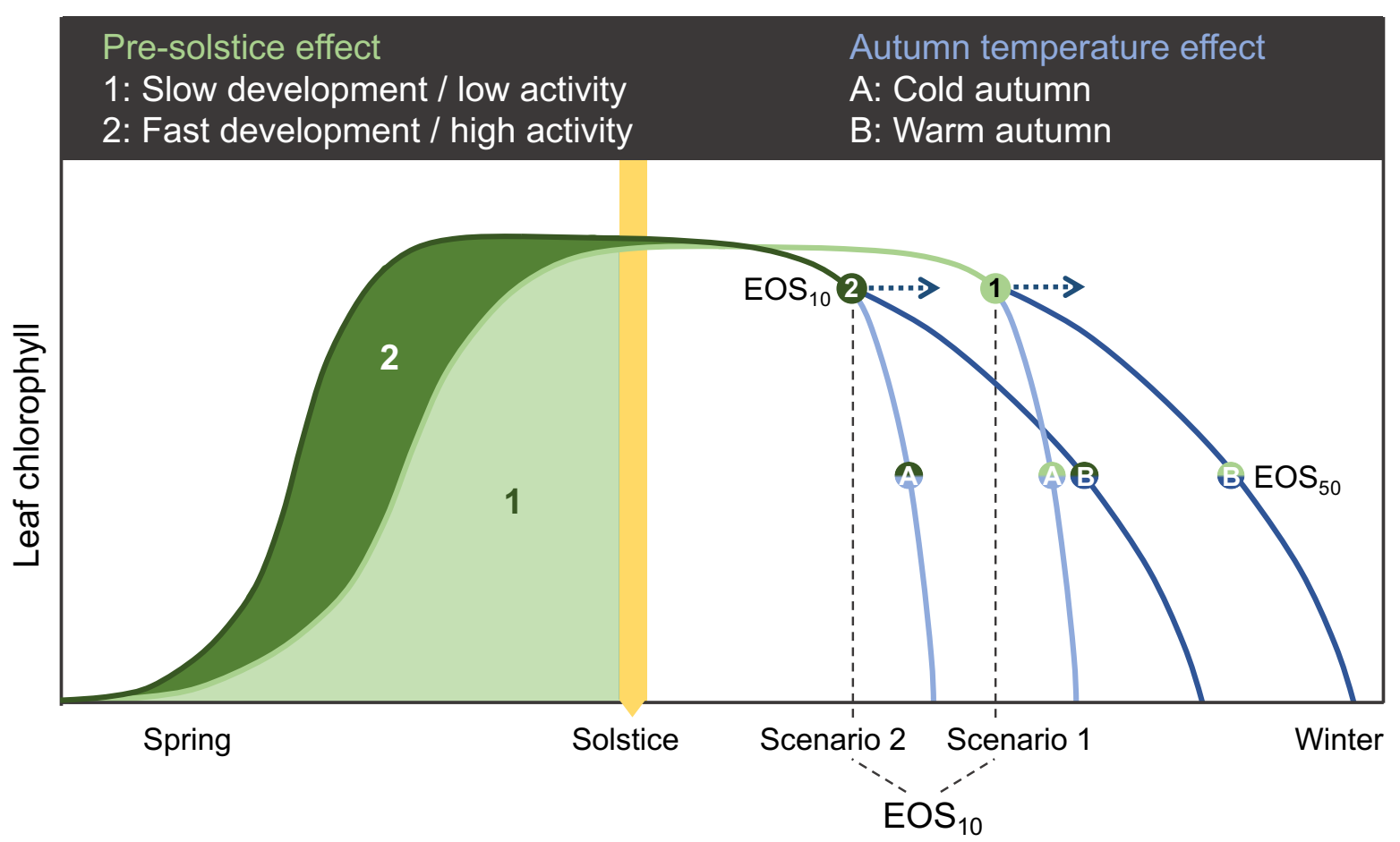

677

678

679

680

681

682

683

684

685

686

687

688

689

690

691

692

693

694

695

696

697
Fig. 1. Conceptual model of autumn phenological responses to pre-solstice and post-solstice growth and temperature (Solstice-as-Phenology-Switch [SPS] hypothesis). The onset of autumnal senescence is estimated in this study as the date when the greenness index last dropped by $>10 \%$ of the seasonal maximum (end-of-season, EOS ${ }_{10}$ ). In northern forests, stem growth and development rates and photosynthetic capacity are highest before the summer solstice and decline with shortening days ${ }^{25-27}$, the adaptive reason being the limited time remaining for tissue maturation and bud set before the first frost. According to the model, inter-annual variation in $\mathrm{EOS}_{10}$ should be a function of pre-solstice growth due to developmental constraints on leaf longevity, with later EOS 10 in years with slow development / low activity before the solstice (scenario 1) and earlier EOS 10 in years with fast development / high activity (scenario 2). The progression of leaf senescence varies with autumn temperature, with faster chlorophyll breakdown in cold-autumn years (scenario A) than in warm-autumn years (scenario B), and the dates of $50 \%$ chlorophyll loss $\left(E S_{50}\right)$ are therefore the combined result of pre- and post-solstice effects. An earlier start of senescence in high-activity years (scenario 2) also predicts that trees will become sensitive to autumn cooling earlier than in low-activity years (see blue arrows). Four fundamental predictions arise from this model that were tested in this study: 1) Enhanced pre-solstice vegetation activity should drive earlier EOS 10 (scenario 1 versus 2). 2) There should be a reversal of growth and temperature effects around the time of the summer solstice. 3) Autumn temperature should affect the speed of senescence and thus have a delaying effect on $\mathrm{EOS}_{50}$ dates (scenario A versus B), but only little effect on $\mathrm{EOS}_{10}$ dates. 4) The date when trees become sensitive to autumn temperature (blue arrows) should have advanced over recent decades because of an earlier onset of senescence $\left(E O S_{10}\right)$. 


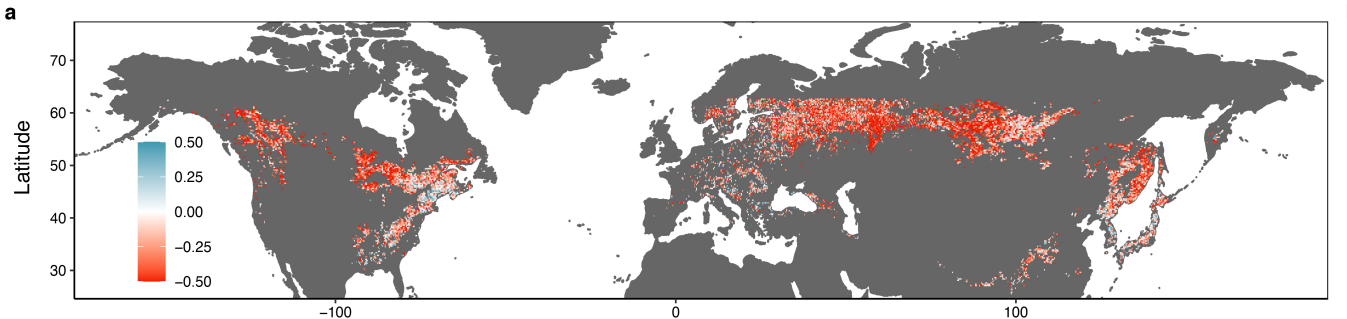

d
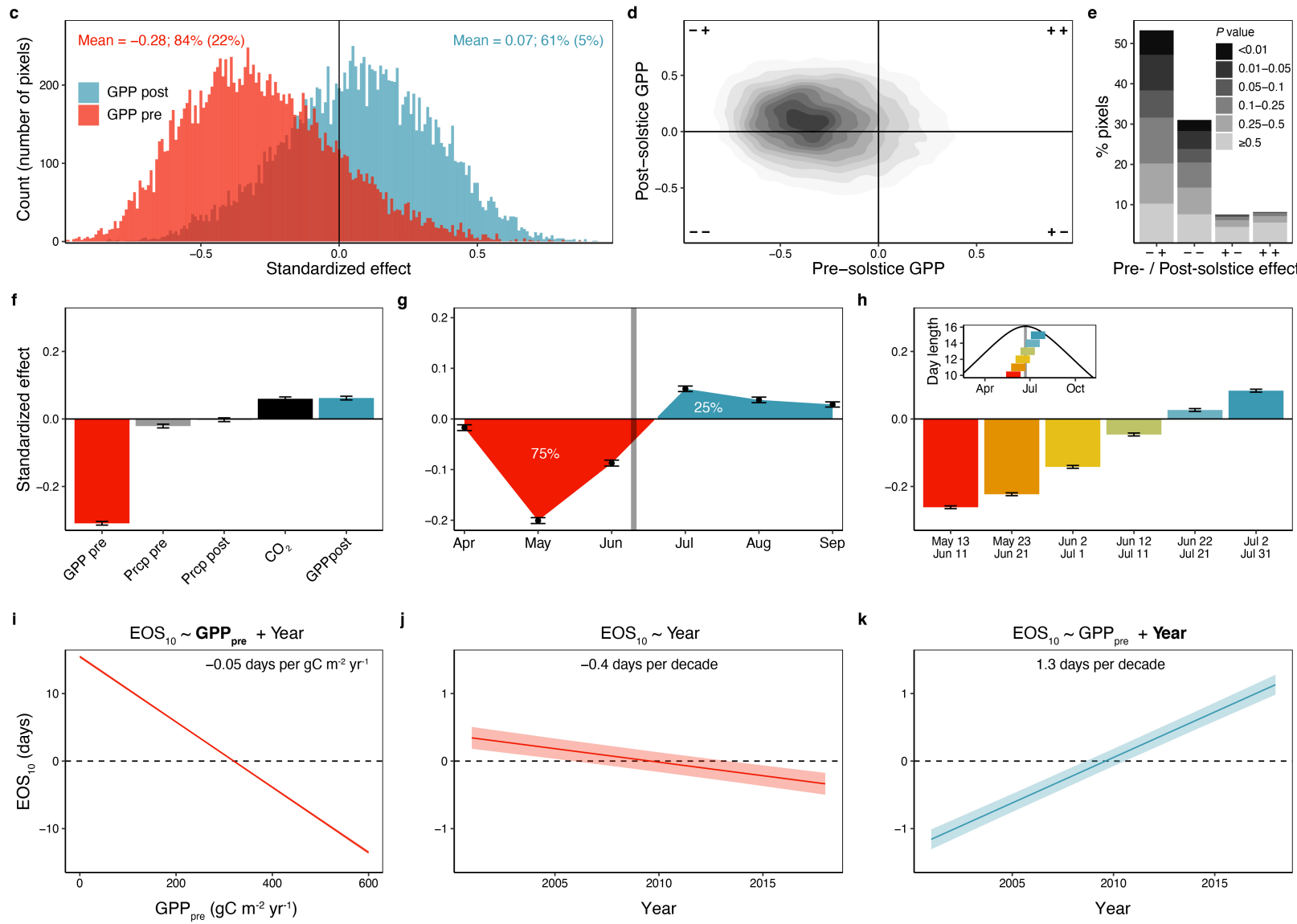

\section{j}
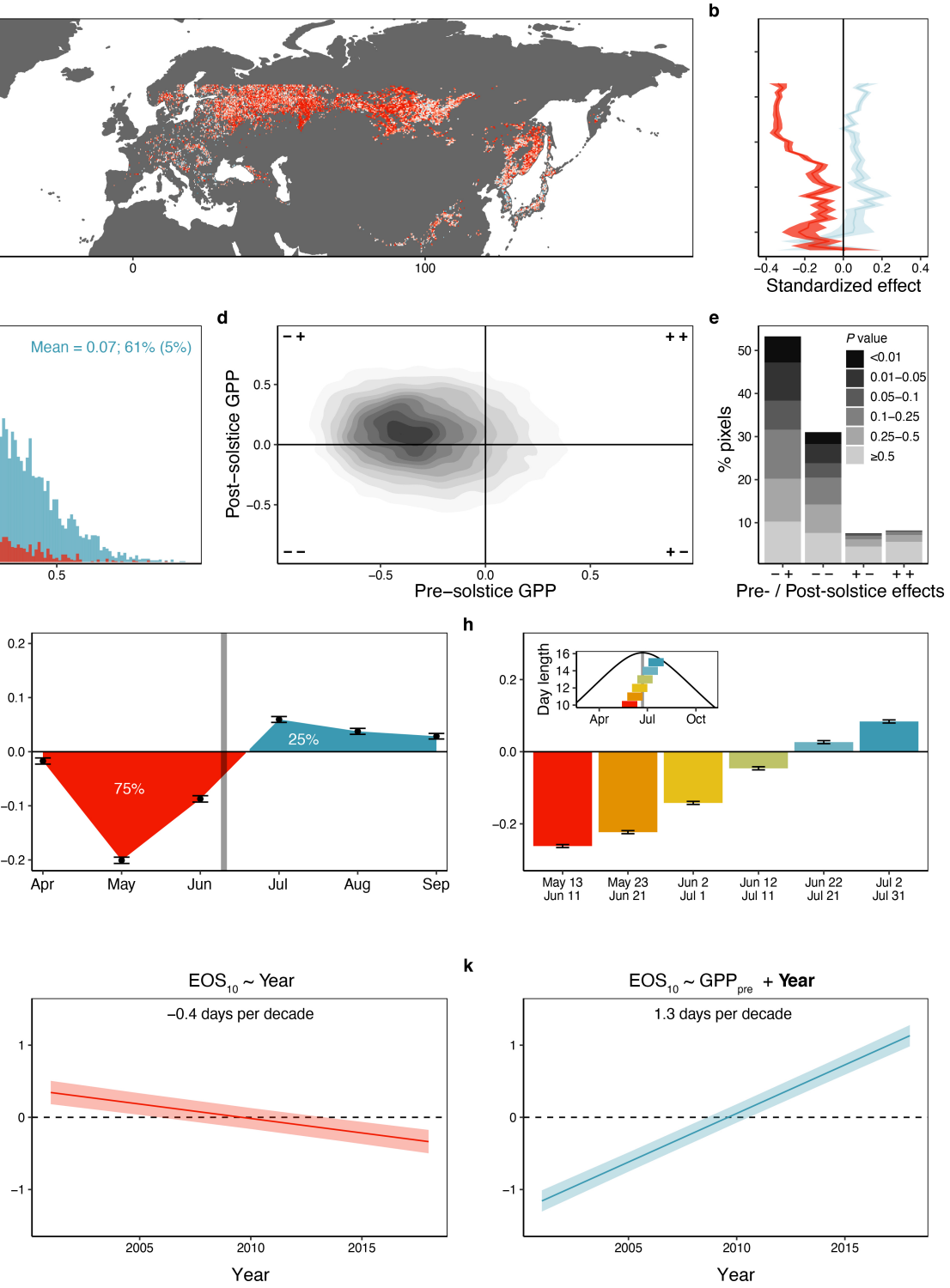

Fig. 2. Satellite observations reveal consistent advances in the onset of senescence $\left(E O S_{10}\right)$ across northern forests in response to enhanced pre-solstice vegetation activity. a, Map showing the standardized effects of pre-solstice (leaf-out to solstice) gross primary productivity (GPP) on EOS 10 timing at $0.25^{\circ}$ resolution from linear models, including pre-solstice GPP and post-solstice (solstice to mean senescence) GPP as predictor variables. Red pixels indicate an earlier EOS ${ }_{10}$ under enhanced pre-solstice GPP, blue pixels indicate a delayed EOS E. $_{10}$ b. Effect size means and $95 \%$ confidence ranges summarized for each degree latitude (pre-solstice effects in red, post-solstice effects in blue). c, The distribution of the pre-solstice and post-solstice GPP effects across all pixels. Mean pre- and post-solstice GPP effect sizes and the percentage of pixels with an advancing pre-solstice GPP effect or delaying post-solstice GPP effect shown as red and blue text, respectively (percentage of significant pixels at $P<0.05$ in brackets). d, Twodimensional density plot of pre- and post-solstice GPP effects. e, Barplot summarizing the effect direction across all analysed pixels. Grey scale indicates significance levels of pre-solstice GPP effects. $\mathbf{f}$, The effects of pre-solstice and post-solstice GPP, pre-solstice (21 March to solstice) and post-solstice precipitation (prcp), and atmospheric $\mathrm{CO}_{2}$. g, Relationship between monthly GPP and $\mathrm{EOS}_{10}$ dates. Percentages reflect the total positive and negative areas under the curve, i.e., the relative advancing versus delaying effects of 
714 seasonal GPP. $\mathbf{h}$, The univariate effects of one-month-long GPP intervals around the summer solstice (May 71513 to June 11, May 23 to June 21, June 2 to July 1, June 12 to July 11 , June 22 to July 21 , and July 2 to 716 July 31 ; see inset). Analyses in $\mathbf{f}-\mathbf{h}$ show effect size means and $95 \%$ confidence ranges from pixel-level 717 linear models with both predictor and dependent variables standardized. $\mathbf{i}-\mathbf{j}$, Mean effects $( \pm 95 \%$ confidence 718 ranges) of pre-solstice GPP and year on EOS 10 anomalies from mixed effects models where pixels are

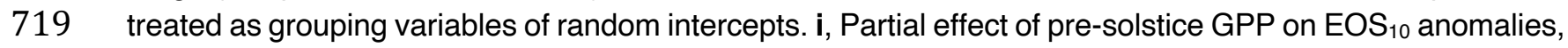
720 including both pre-solstice GPP and year as fixed effects. j, Temporal trend in $\mathrm{EOS}_{10}$ anomalies with year 721 as single fixed effect. $\mathbf{k}$, Partial effect of year on $\mathrm{EOS}_{10}$ anomalies, where both pre-solstice GPP and year 722 are treated as fixed effects.

723 


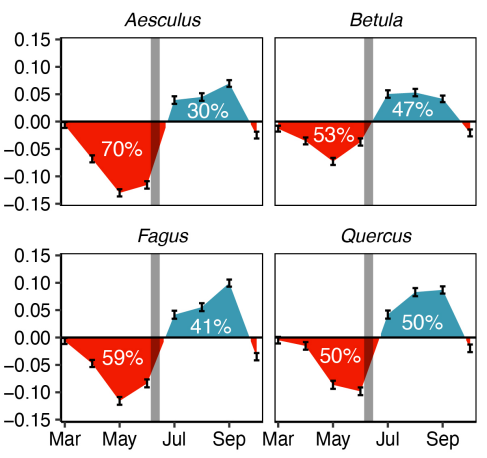

d

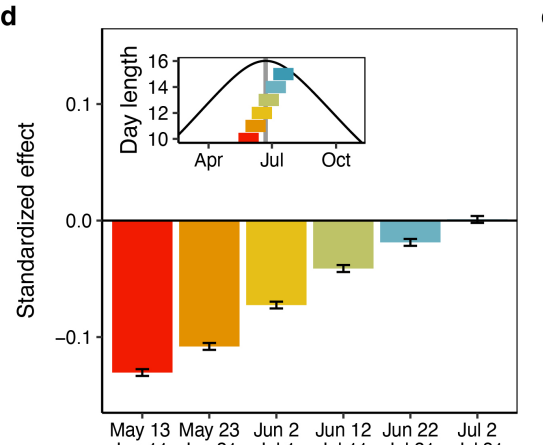

$\mathbf{g}$

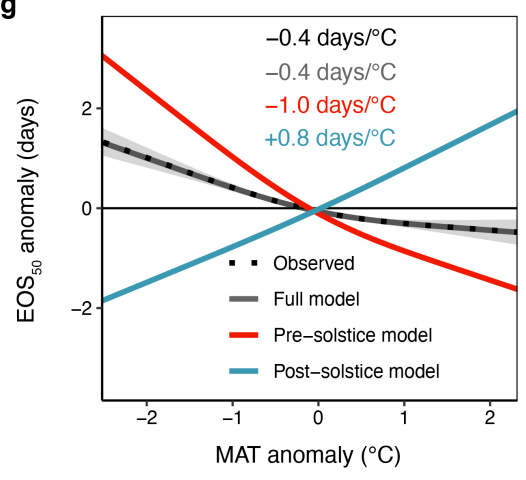

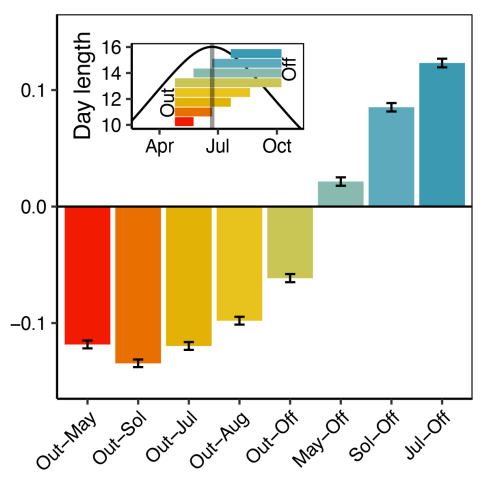

h

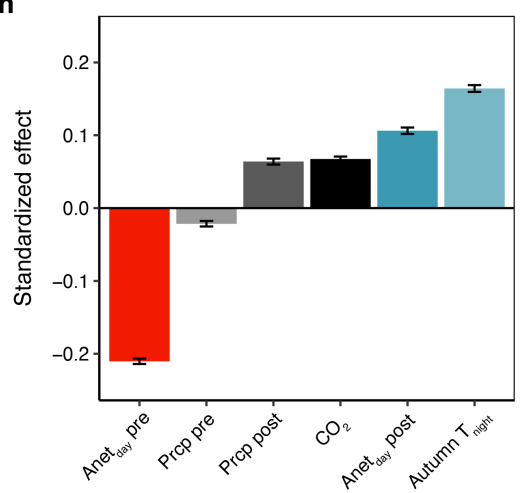

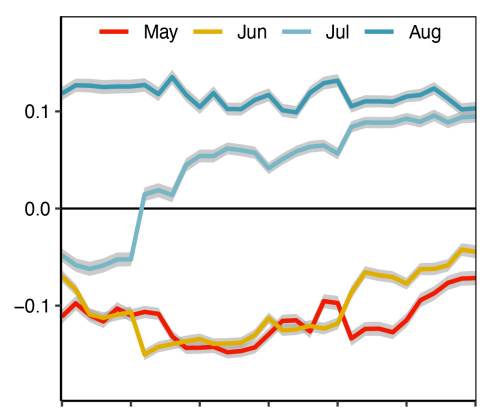

C

f

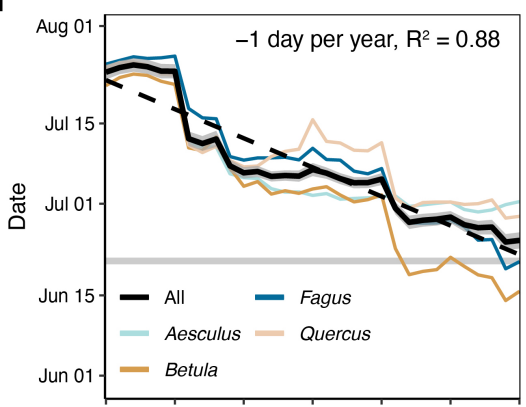

724

725

726

727

728

729

730

731

732

733

734

735

736

737

738

Fig. 3. Relationships between seasonal photosynthesis and the timing of mid-senescence $\left(E S_{50}\right)$ from European long-term observations (PEP725 data). a, Effects of monthly (March to October) photosynthesis on $\mathrm{EOS}_{50}$ dates. Percentages reflect the relative advancing versus delaying effects of seasonal photosynthesis on EOS ${ }_{50}$ dates. b, Species-level results with percentages as in panel a. c, Movingwindow analysis, showing the effects of May (leaf-out to 31 May), June, July, and August/September (1 August to 30 September) photosynthesis for each 20-year time period from 1966 to 2015. d, Effects of onemonth-long photosynthesis intervals around the summer solstice (May 13 to June 11, May 23 to June 21, June 2 to July 1 , June 12 to July 11 , June 22 to July 21 , and July 2 to July 31 ; see inset), including the respective photosynthesis interval and autumn night-time temperature (Autumn $T_{\text {night }}$ ) as fixed effects. e, The effects of cumulative photosynthesis from leaf-out to May 22 (Out-May), leaf-out to solstice (Out-Sol), leaf-out to July 21 (Out-Jul), leaf-out to August 20 (Out-Aug), leaf-out to mean EOS ${ }_{50}$ (Out-Off), May 22 to mean EOS 50 (May-Off), solstice to mean EOS 50 (Sol-Off), and July 21 to mean EOS 50 (Jul-Off), including the respective photosynthesis interval as single fixed effect. $\mathbf{f}$, Moving-window analysis, showing the 'reversal' dates when the photosynthesis effect switches from negative to positive for each 20-year time 
739 period from 1966 to 2015 (based on monthly correlations, see panels a-c). On average, the reversal date

740 advanced by 1 day per year. $\mathbf{g}-\mathbf{i}$, The effects of pre-solstice (leaf-out to solstice) and post-solstice (solstice

741 to mean $\mathrm{EOS}_{50}$ ) photosynthesis, pre-solstice (21 March to solstice) and post-solstice precipitation,

742 atmospheric $\mathrm{CO}_{2}$, and Autumn $\mathrm{T}_{\text {night. }} \mathbf{g}$, Model predictions in response to mean annual temperature (MAT)

743 anomalies (black dashed line: observed trend; black solid line: full model prediction including pre- and post-

744 solstice effects; red line: model prediction including only pre-solstice photosynthesis and precipitation and

$745 \mathrm{CO}_{2}$ as predictors; blue line: model prediction including only post-solstice photosynthesis and precipitation,

$746 \mathrm{CO}_{2}$, and Autumn $\mathrm{T}_{\text {night }}$ ). h, Standardised effects. i, 20-year moving-window analysis of the effects (colours

747 as in panel $\mathbf{h}$ ). Analyses show effect size means \pm 2 s.e. from linear mixed models, including time series

748 and species $(\mathbf{a}, \mathbf{c}-\mathbf{i})$ or only time series $(\mathbf{b}, \mathbf{f})$ as random effects, with both predictor and dependent variables

749 standardized.

750 


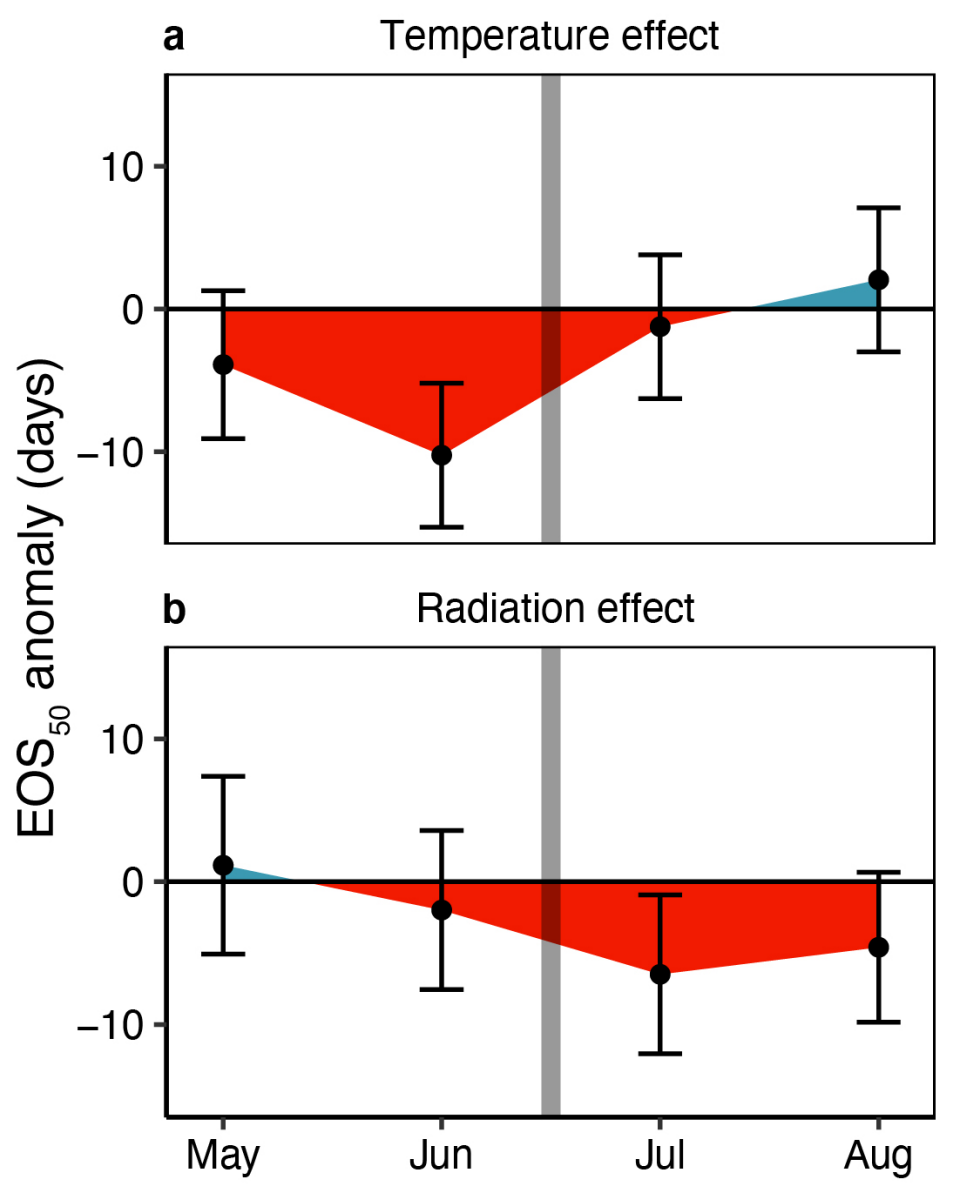

Fig. 4. The seasonal effects of temperature (a) and shading (b) on autumn phenology in European 754 beech from experiment 1. Effects of monthly (May to August) cooling (a) and shading (b) treatments on EOS $_{50}$ dates. Analyses show effect size means $\pm 95 \%$ confidence intervals from linear models, including treatment as fixed effect. Note that effect directions were reversed to facilitate comparison with Figs. 2 and 3 , i.e., negative values indicate EOS $_{50}$ advances under warmer (a) or brighter (b) conditions. See methods 758 
a

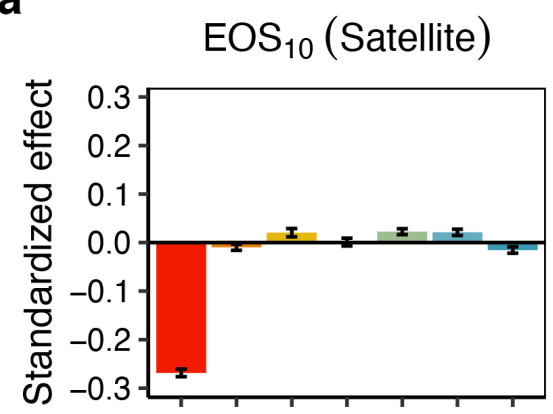

b

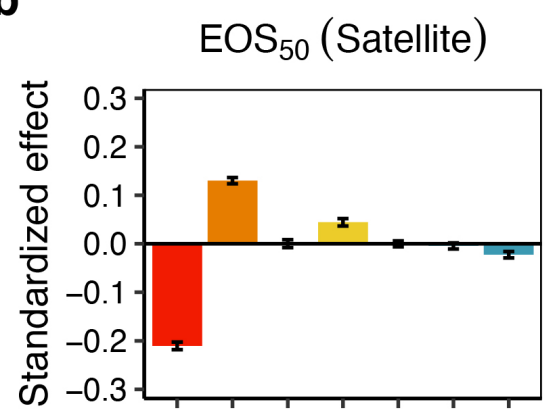

C

$\mathrm{EOS}_{50}(\mathrm{PEP725}$ data)

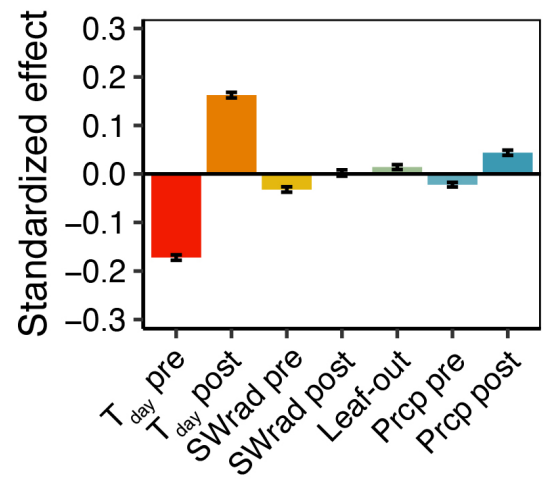

759

760

Fig. 5. The effects of pre- and post-solstice temperature, radiation, water availability and spring leaf-

761 out dates on inter-annual variation in the timing of EOS ${ }_{10}(a)$ and $E O S_{50}(b, c)$. We ran linear models

762 including mean day-time temperature ( $\left.T_{\text {day }}\right)$ and short-wave radiation (SWrad) and the sums of precipitation

763 (prcp) from March 20 to June 21 (pre-solstice) and from June 22 to the mean EOS date of each time series

764 (post-solstice) as well as spring leaf-out dates as predictor variables. Models were run at the pixel-level (a,

765 b) or individual-level (c) and the mean effects ( $\pm 95 \%$ confidence intervals) are shown. All variables were standardized to allow for effect size comparison.

767 
768 Extended Data Figures

769 


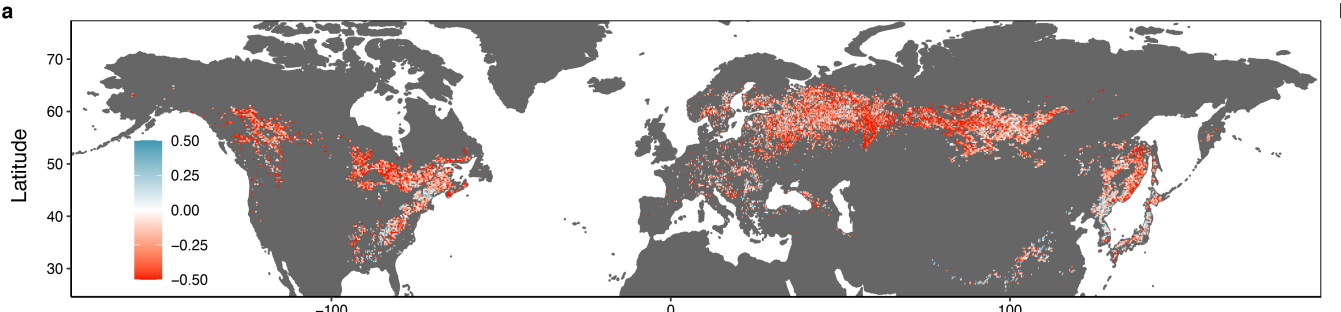

d
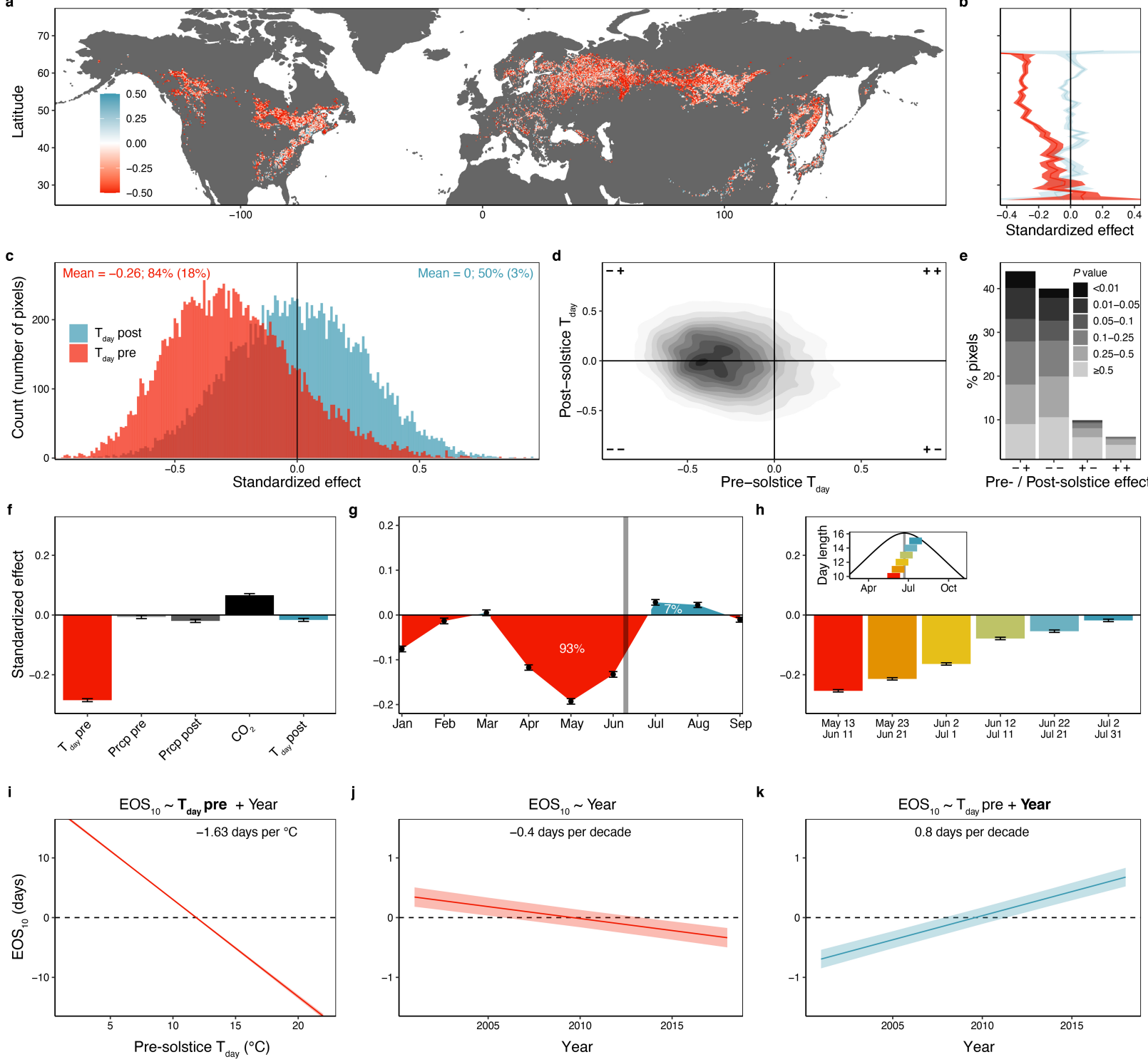

k

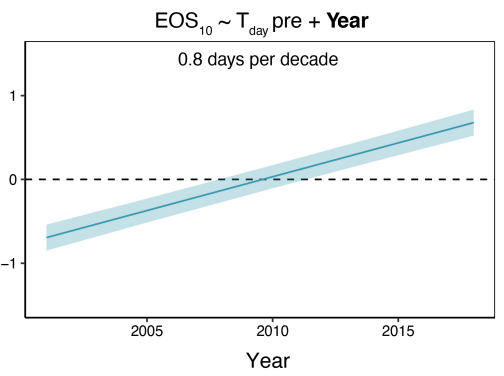

Extended Data Fig. 1. The seasonal effects of day-time temperature $\left(T_{\text {day }}\right)$ on inter-annual variation in senescence onset (EOS ${ }_{10}$ dates) [same as Fig. 2 but using $\mathbf{T}_{\text {day }}$ as predictor variable]. a, Map showing the standardized effects of pre-solstice (leaf-out to solstice) $\mathrm{T}_{\text {day }}$ on $\mathrm{EOS}_{10}$ timing at $0.25^{\circ}$ resolution from linear models, including pre-solstice $\mathrm{T}_{\text {day }}$ and post-solstice (solstice to mean senescence) $T_{\text {day }}$ as predictor variables. Red pixels indicate an earlier $E O S_{10}$ under enhanced pre-solstice $T_{\text {day }}$, blue pixels indicate a delayed $\mathrm{EOS}_{10}$. b. Effect size means and 95\% confidence ranges summarized for each degree latitude (pre-solstice effects in red, post-solstice effects in blue). c, The distribution of the pre-solstice and post-solstice $T_{\text {day }}$ effects across all pixels. Mean pre- and postsolstice $T_{\text {day }}$ effect sizes and the percentage of pixels with a negative pre-solstice $T_{\text {day }}$ or positive post-solstice $T_{\text {day }}$ effect (percentage of significant pixels at $P<0.05$ in brackets) shown as red and blue text, respectively. d, Two-dimensional density plot of pre- and postsolstice $T_{\text {day }}$ effects. e, Barplot summarizing the effect direction across all analysed pixels. Grey scale indicates significance levels of pre-solstice $T_{\text {day }}$ effects. $f$, The effects of pre-solstice and post-solstice $T_{\text {day }}$, pre-solstice (21 March to solstice) and post-solstice precipitation, and atmospheric $\mathrm{CO}_{2}$. g, Relationship between monthly $\mathrm{T}_{\text {day }}$ and $\mathrm{EOS}_{10}$ dates. Percentages reflect the total positive and negative areas under the curve, i.e., the relative advancing versus delaying effects of seasonal $T_{\text {day. }} \mathbf{h}$, The univariate effects of onemonth-long $T_{\text {day }}$ intervals around the summer solstice (May 13 to June 11, May 23 to June 21, June 2 to July 1, June 12 to July 11 , June 22 to July 21, and July 2 to July 31; see inset). Analyses in $\mathbf{f}-\mathbf{h}$ show effect size means and $95 \%$ confidence ranges from pixellevel linear models with both predictor and dependent variables standardized. $\mathbf{i}-\mathbf{j}$, Mean effects $( \pm 95 \%$ confidence ranges) of presolstice $T_{\text {day }}$ and year on $E O S_{10}$ anomalies from mixed effects models where pixels are treated as grouping variables of random intercepts. i, Partial effect of pre-solstice $T_{\text {day }}$, including both pre-solstice $T_{\text {day }}$ and year as fixed effects. $\mathbf{j}$, Temporal trend in EOS 10 anomalies with year as single fixed effect. $\mathbf{k}$, Partial effect of year, where both pre-solstice $T_{\text {day }}$ and year are treated as fixed effects. 

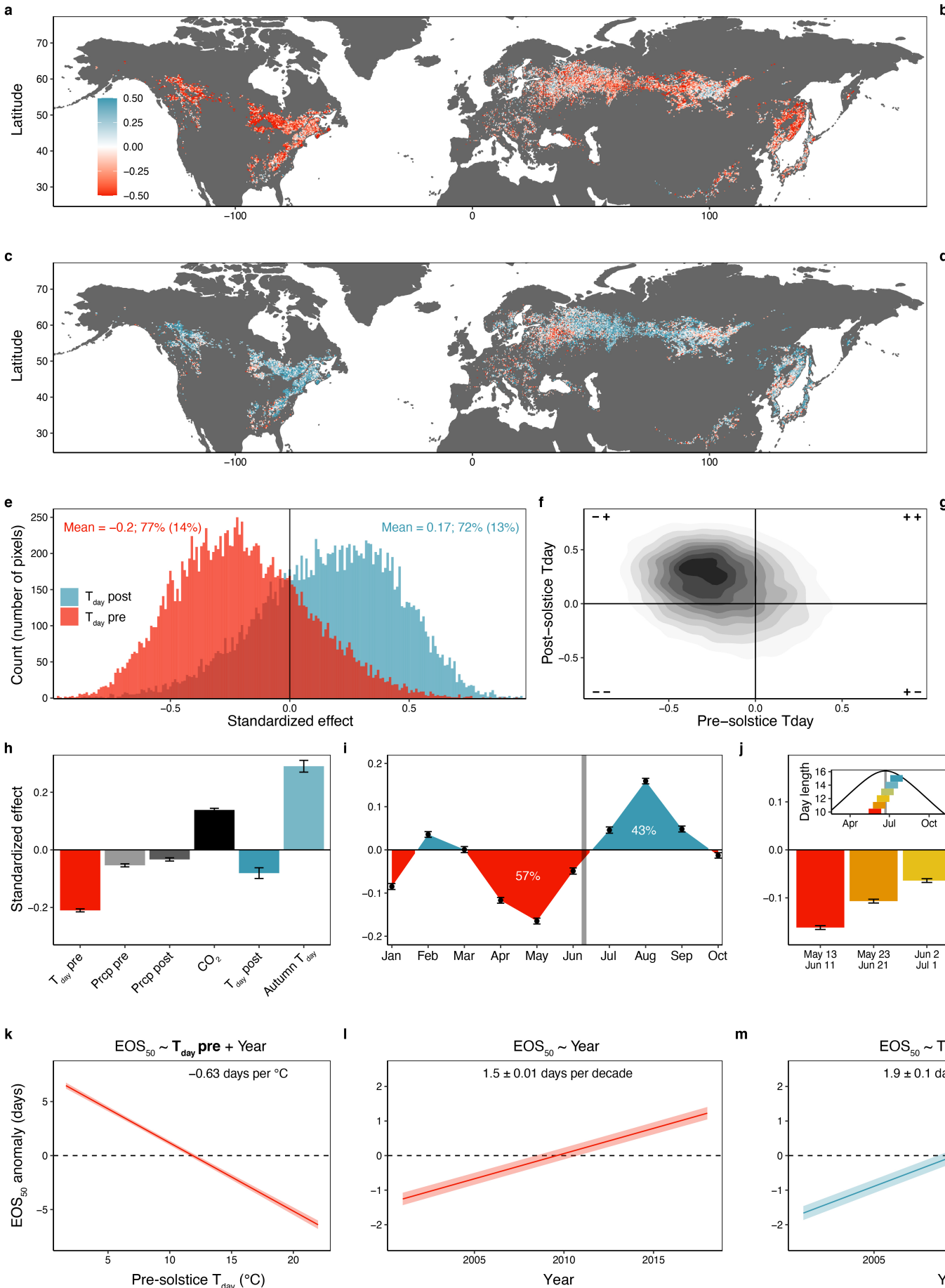

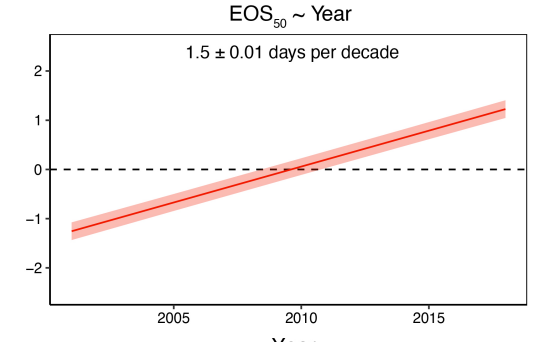

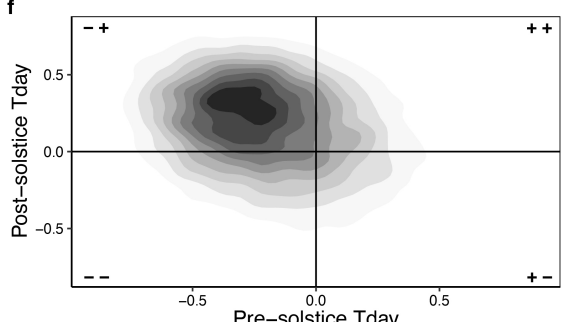

j

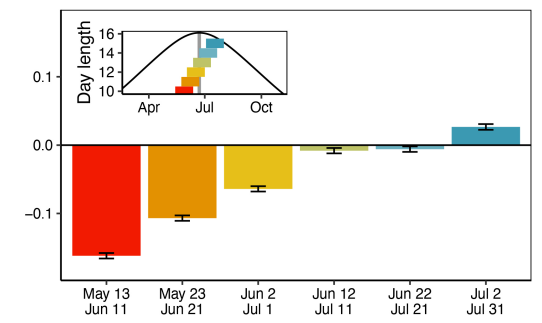

Extended Data Fig. 2. The seasonal effects of $T_{\text {day }}$ on inter-annual variation in mid-senescence (EOS ${ }_{50}$ dates). a, $\mathbf{c}$, Maps showing the standardized effects of pre-solstice $\mathrm{T}_{\text {day }}$ (leaf-out to solstice; $\mathbf{a}$ ) and post-solstice $\mathrm{T}_{\text {day }}$ (solstice to mean EOS $\mathrm{S}_{50}$, $\mathbf{b}$ ) on EOS EO $_{50}$ timing at $0.25^{\circ}$ resolution from linear models, including pre-solstice $T_{\text {day }}$ and post-solstice $T_{\text {day }}$ as predictor variables. Red pixels indicate an earlier $\mathrm{EOS}_{50}$ under higher pre-solstice or post-solstice $\mathrm{T}_{\text {day }}$, respectively, blue pixels indicate a delayed EOS $\mathrm{E}_{50}$. b, d, Effect size

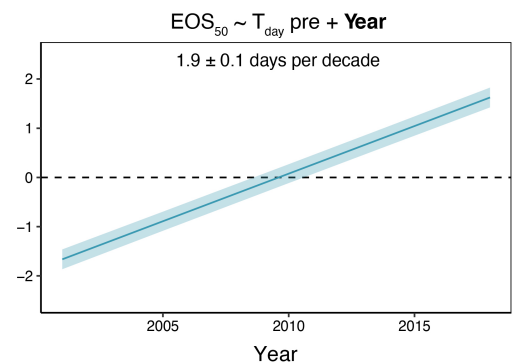
means and $95 \%$ confidence ranges summarized for each degree latitude (pre-solstice effects in red (b), post-solstice effects in blue (d)). e, The distribution of the pre-solstice and post-solstice $T_{\text {day }}$ effects across all pixels. Mean pre- and post-solstice $T_{\text {day }}$ effect sizes and the percentage of pixels with a negative pre-solstice $T_{\text {day }}$ or positive post-solstice $T_{\text {day }}$ effect (percentage of significant pixels at $P$ $<0.05$ in brackets) shown as red and blue text, respectively. f. Two-dimensional density plot of pre- and post-solstice $T_{\text {day }}$ effects. $\mathbf{g}$, Barplot summarizing the effect direction across all analysed pixels. Grey scale indicates significance levels of pre-solstice $T_{\text {day }}$ effects. 
801

802

803

804

805

806

807

808

809
$\mathrm{CO}_{2}$, and autumn $\mathrm{T}_{\text {day. }} \mathbf{i}$, Relationship between monthly $\mathrm{T}_{\text {day }}$ and $\mathrm{EOS}_{50}$ dates. Percentages reflect the total positive and negative areas under the curve, i.e., the relative advancing versus delaying effects of seasonal $T_{\text {day. }} \mathbf{j}$, The univariate effects of one-month-long $T_{\text {day }}$ intervals around the summer solstice (May 13 to June 11, May 23 to June 21, June 2 to July 1, June 12 to July 11 , June 22 to July 21 , and July 2 to July 31; see inset). Analyses in $\mathbf{h}-\mathbf{j}$ show effect size means and $95 \%$ confidence ranges from pixel-level linear models with both predictor and dependent variables standardized. $\mathbf{k}-\mathbf{m}$, Mean effects ( $\pm 95 \%$ confidence ranges) of pre-solstice $T_{\text {day }}$ and year on $\mathrm{EOS}_{50}$ anomalies from mixed effects models where pixels are treated as grouping variables of random intercepts. $\mathbf{k}$, Partial effect of pre-solstice $T_{\text {day, }}$, including both pre-solstice $T_{\text {day }}$ and year as fixed effects. I, Temporal trend in $\mathrm{EOS}_{50}$ anomalies with year as single fixed effect. $\mathbf{m}$, Partial effect of year, where both pre-solstice $T_{\text {day }}$ and year are treated as fixed effects. 
a
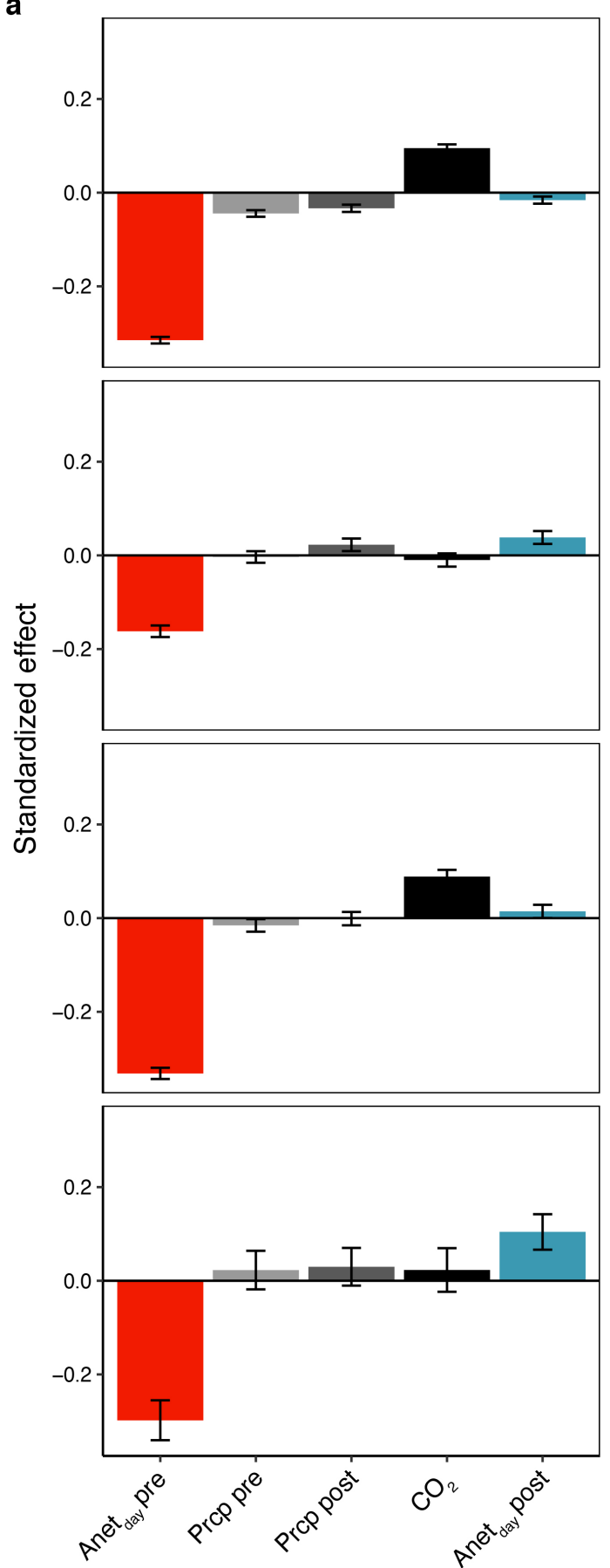

b
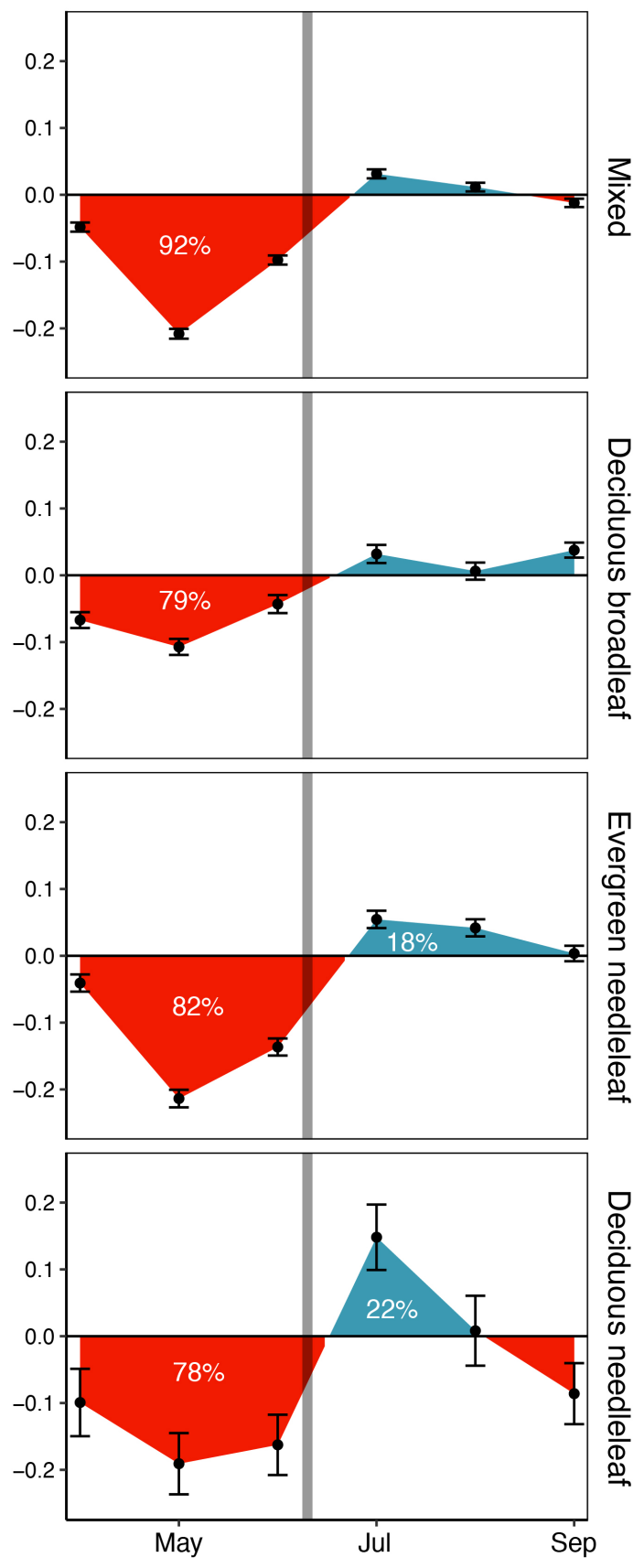

810

Extended Data Fig. 3. Relationships between seasonal productivity (Anet day) and the timing of senescence onset $\left(E O S_{10}\right)$ within mixed, deciduous broadleaf, deciduous needleleaf, and evergreen needleleaf forests. a, The effects of pre-solstice and post-solstice Anet day, pre-solstice (21 March to solstice) and post-solstice precipitation, and atmospheric $\mathrm{CO}_{2}$ on $\mathrm{EOS}_{10}$ dates. $\mathbf{b}$, Relationship between monthly Anet day $_{\text {and }}$ and $\mathrm{EOS}_{10}$ dates. Percentages reflect the total positive and negative areas under the curve, i.e., the relative advancing versus delaying effects of seasonal Anetday. 


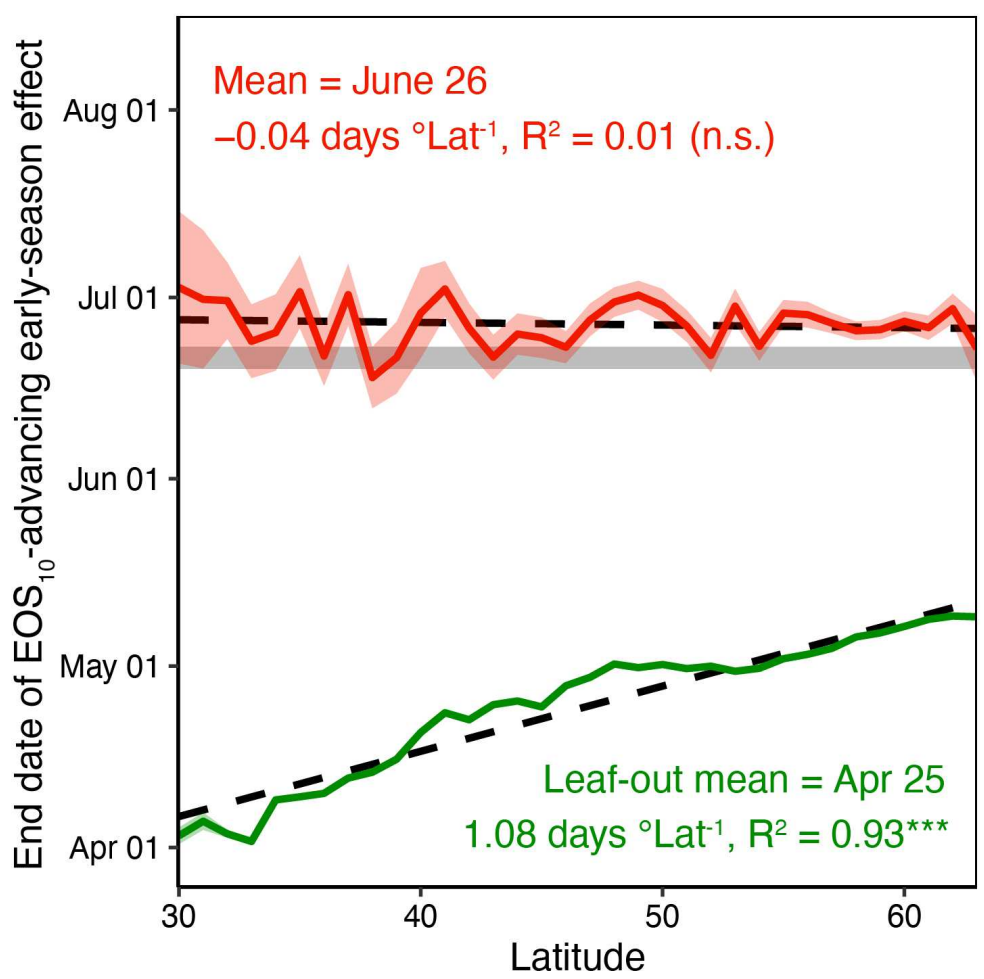

818

819

820 Extended Data Fig. 4. End date (red line) of the early-season growth period exhibiting an advancing 821 effect on the onset of senescence $\left(\right.$ EOS $\left._{10}\right)$. Pixel-level linear regression models were applied to test for 822 the effect of gross primary productivity (GPP) on EOS ${ }_{10}$ dates. GPP summation always started at leaf-out 823 and ended at different dates, using 30-day steps. For each pixel, we then obtained the period that showed 824 the most negative effect on $\mathrm{EOS}_{10}$ dates. The red line shows the end date of this period along latitude, 825 whereby pixel-level estimates were averaged for each degree latitude. Across the entire latitudinal range, 826 the end date of the early-season effect consistently occurred right after the summer solstice at June 26. 827 Red text shows the mean date as well as the slope and $\mathrm{R}^{2}$ for the correlation between latitude and the end 828 date of the early-season effect $\left(R^{2}=0.01\right)$. The green line represents the average spring leaf-out dates for 829 each degree latitude, showing an average delay in leaf-out of 1.08 days per each degree increase in 830 northern latitude $\left(R^{2}=0.93\right)$. Grey line represents the summer solstice $(21$ June). Non-significant (n.s.): $P$ $831>0.05 ;{ }^{* \star *} \mathrm{P}<0.001$. 
a
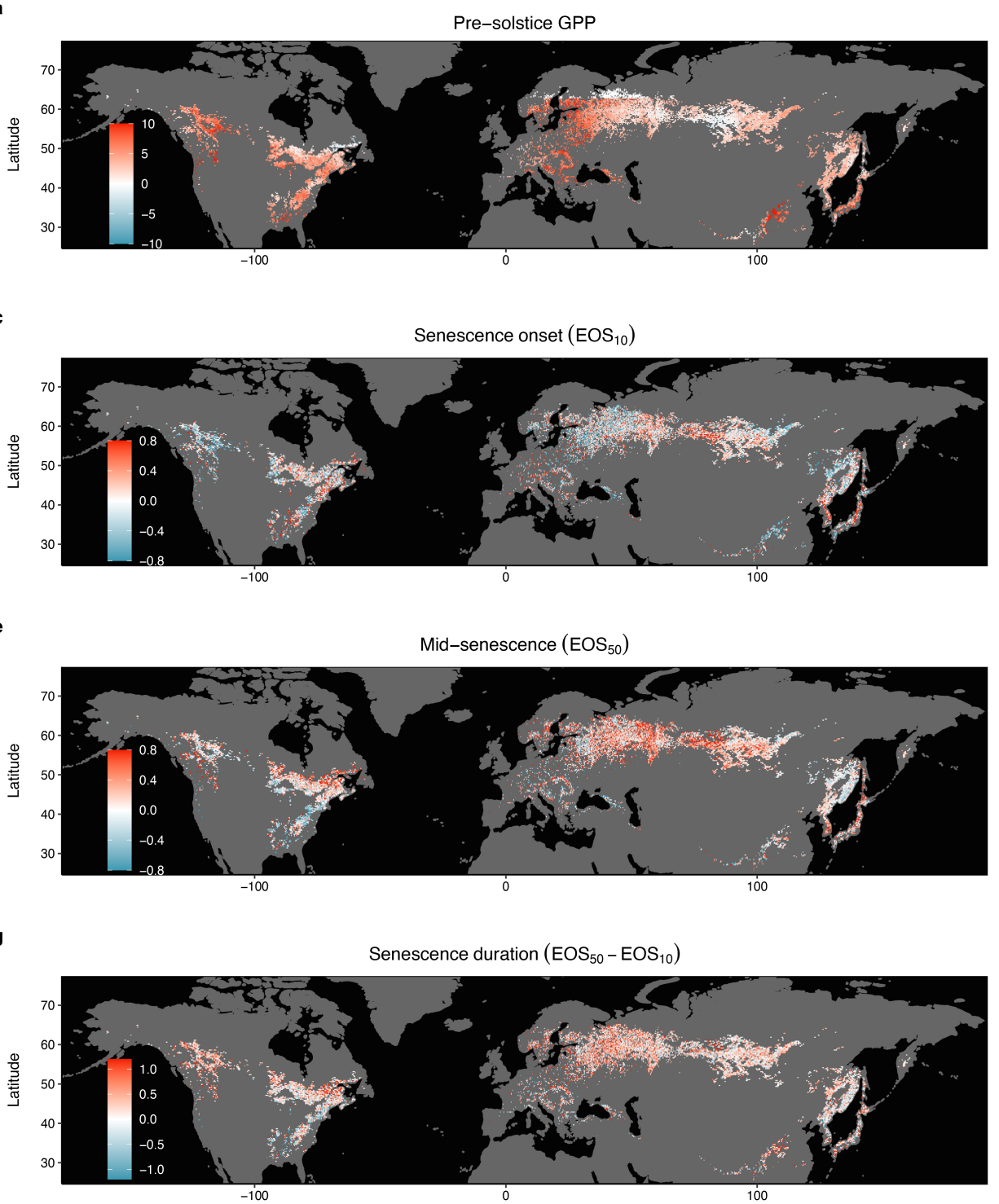

i

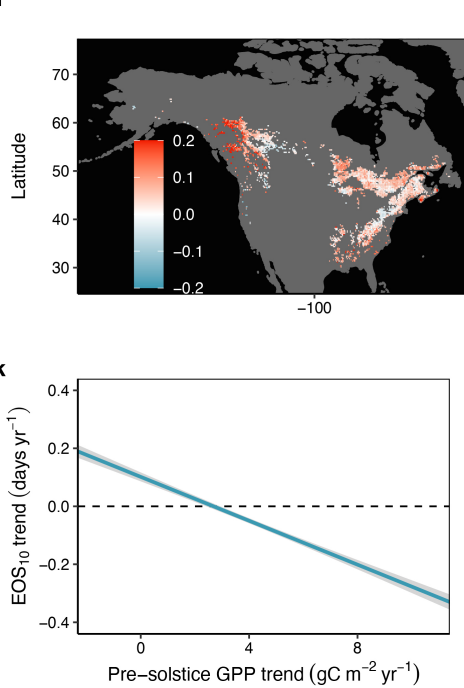

Autumn temperature
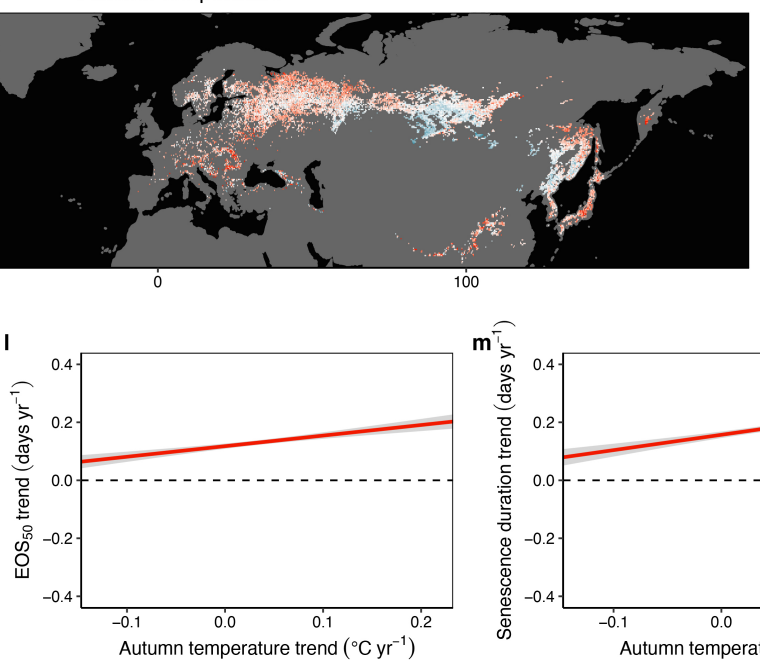

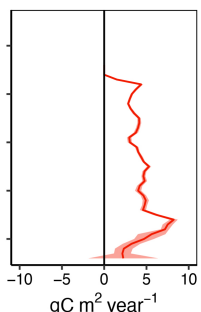

d
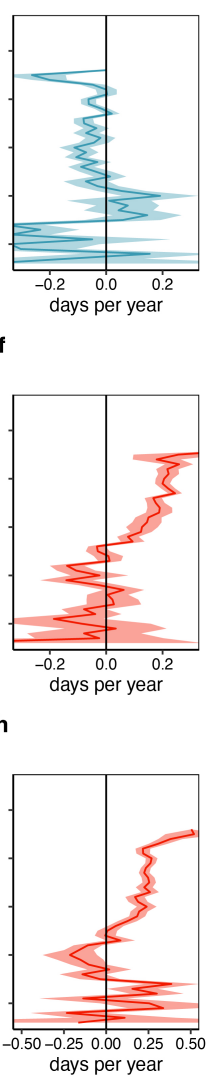

j
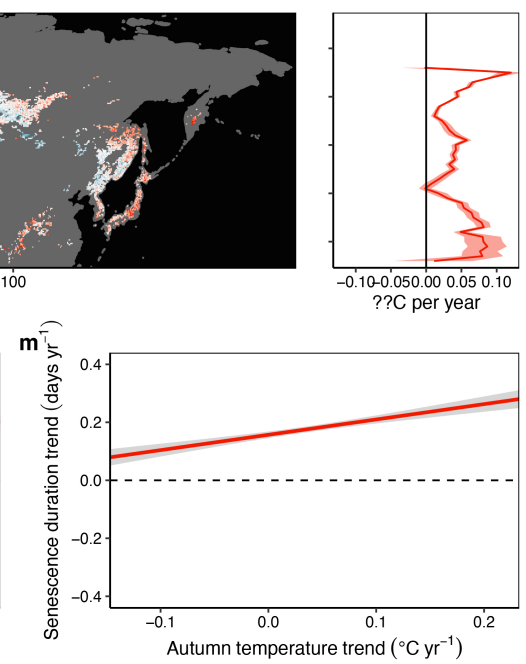
834 Extended Data Fig. 5. Temporal trends in (a) pre-solstice gross primary productivity [GPP], (c) 835 senescence onset $\left(E \mathrm{~S}_{10}\right)$ dates, $(\mathrm{e})$ mid-senescence $\left(\mathrm{EOS}_{50}\right)$ dates, $(\mathrm{g})$ senescence duration $\left(\mathrm{EOS}_{10^{-}}\right.$ 836 to-EOS ${ }_{50}$ ) and (i) autumn temperature at $0.25^{\circ}$ resolution for the $2001-2018$ period. Red pixels indicate

837 delays in $\mathrm{EOS}_{10 / 50}$ dates $(\mathbf{c}, \mathbf{e})$, a longer duration of the senescence period $(\mathbf{g})$, or increases in pre-solstice

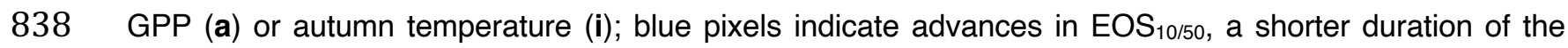
839 senescence period, or decreases in pre-solstice GPP and autumn temperature. $\mathbf{b}, \mathbf{d}, \mathbf{f}, \mathbf{h}, \mathbf{j}$, Trend means 840 and $95 \%$ confidence ranges summarized for each degree latitude. All trends were inferred from linear, 841 univariate models. $\mathbf{k}-\mathbf{m}$, Linear regression ( $\pm 95 \%$ confidence interval) between pixel-level temporal trends

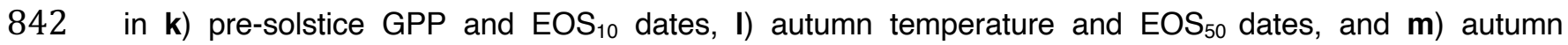
843 temperature and senescence duration. Areas with large increases in pre-solstice GPP also show larger 844 advances in $\mathrm{EOS}_{10}$ dates, whereas areas in which GPP has been declining tend to show delays in EOS $\mathrm{S}_{10}$ 845 (k). Areas with large increases in autumn temperature show larger delays in $\mathrm{EOS}_{50}$ dates (I) and, 846 accordingly, a prolongation of senescence duration $(\mathbf{m})$. Autumn temperature represents the mean day-time 847 autumn temperature for the autumn period that was most highly correlated with EOS $\mathrm{S}_{50}$ dates (Autumn $\mathrm{T}_{\text {day; }}$ 848 see Autumn temperature intervals in methods section). 


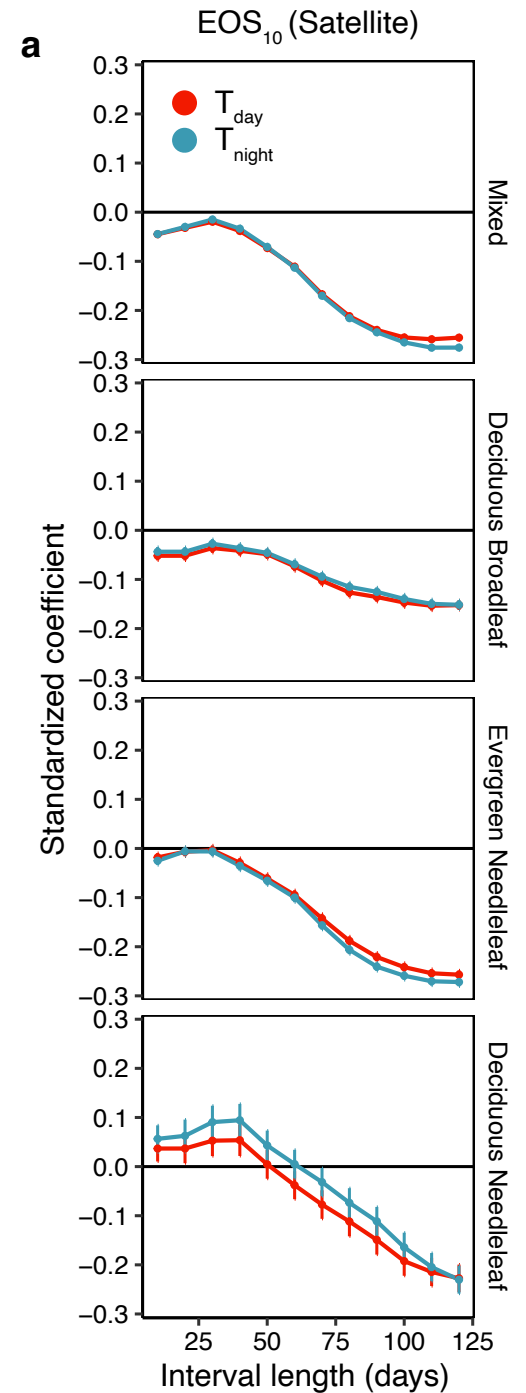

b

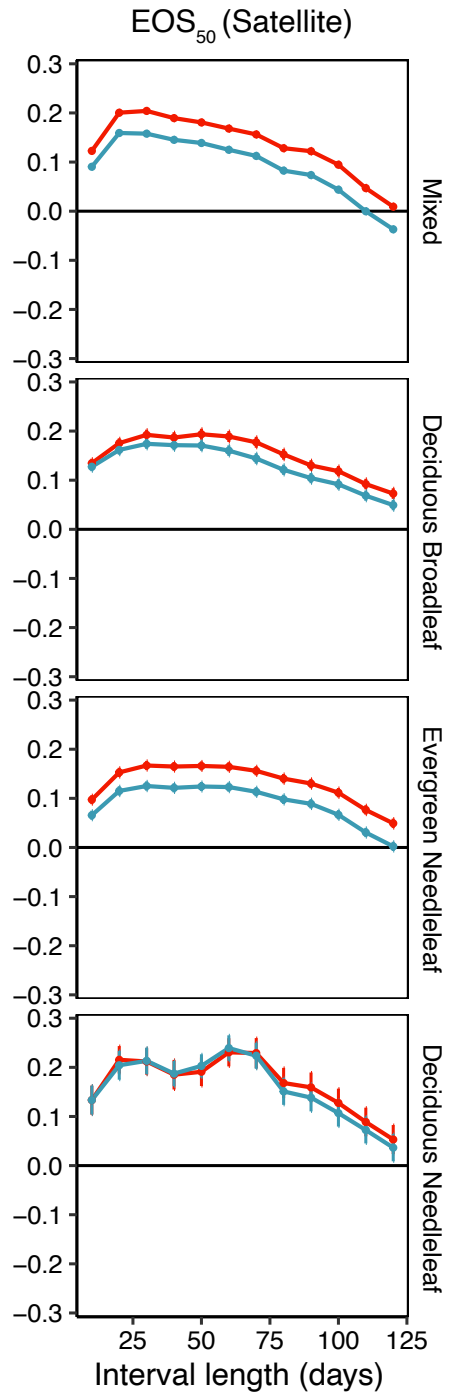

C
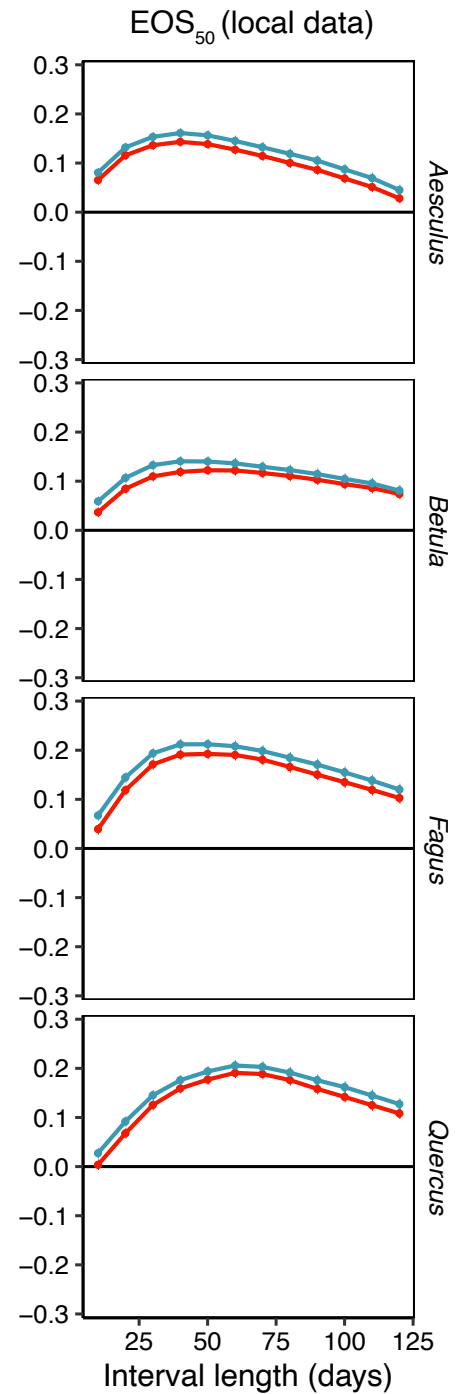

850

851

852

853

854

855

856

857

858

859

860

861
Extended Data Fig. 6. The effect of autumn temperature on $\operatorname{EOS}_{10}(a)$ and $\operatorname{EOS}_{50}$ dates (b,c). Standardized regression coefficients for the univariate correlations between autumn temperature intervals and EOS dates. Temperature intervals refer to the mean night-time ( $\left.T_{\text {night }}\right)$ or day-time $\left(T_{\text {day }}\right)$ temperatures for the periods 10 to 120 days before the mean $\mathrm{EOS}_{10}$ or $\mathrm{EOS}_{50}$ date at each time series. Models were run at the pixel-level $(\mathbf{a}, \mathbf{b})$ or individual-level $(\mathbf{c})$ and the mean time series-level effects $( \pm 95 \%$ confidence intervals) are shown separately for mixed, deciduous broadleaf, evergreen needleleaf, and deciduous needleleaf forests for the satellite-based analyses and for the four European species included in the PEP725 dataset. 
a

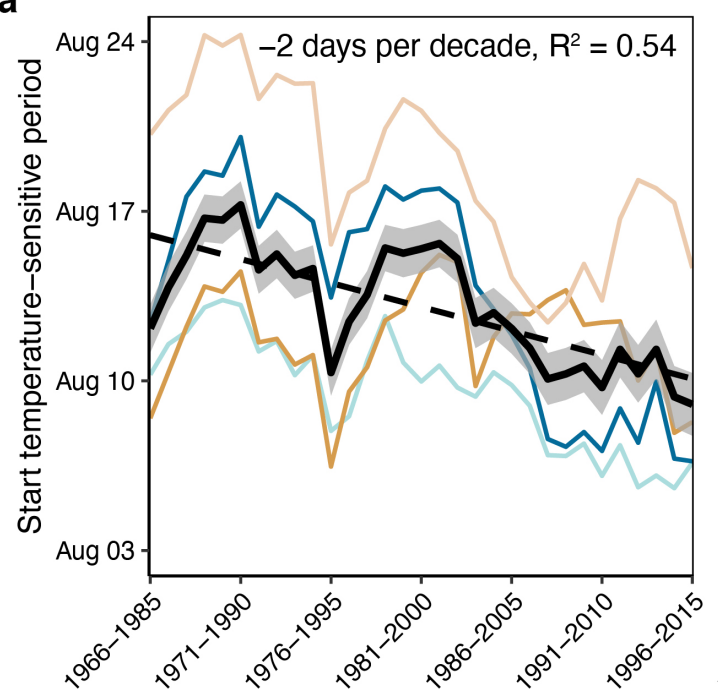

b

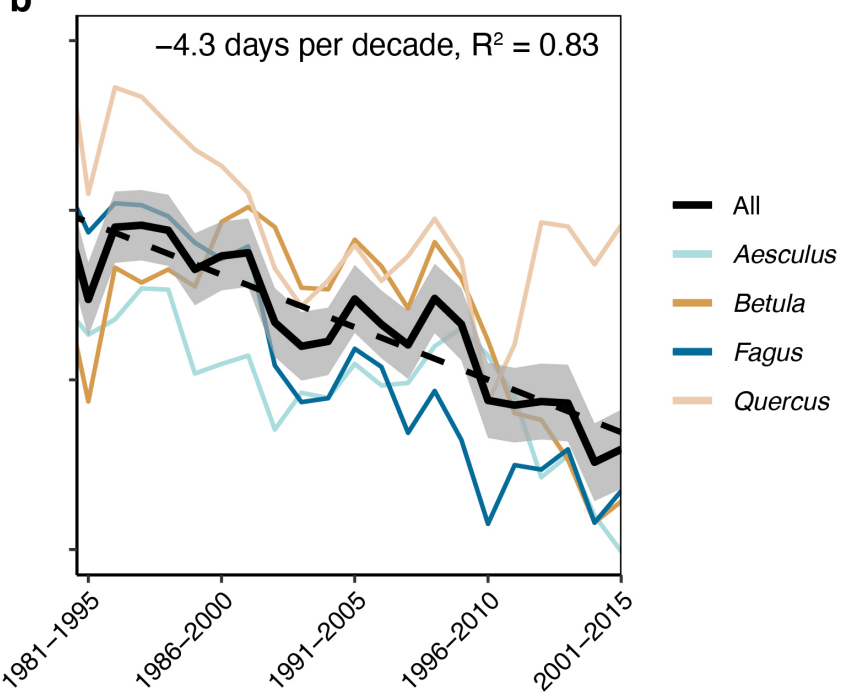

862

863

864

865

866

867

868

869

870
Extended Data Fig. 7. Moving-window analysis based on European long-term observations (PEP725 data), showing the average dates when trees became sensitive to autumn temperatures for each 20 year time period from 1966 to 2015 (a) and for each 15-year time period from 1981 to 2015 (b). Coloured lines indicate species-specific results, the black line represents the mean $( \pm 95 \%$ confidence interval) across all species, the black dashed line indicates the trend from a linear model. This trend line shows that the start date of the temperature-sensitive autumn period advanced by $2.0 \pm 0.7$ days (mean \pm $95 \%$ confidence interval) per decade (a) or by $4.3 \pm 0.9$ days per decade for the 1981-2015 period (b). 


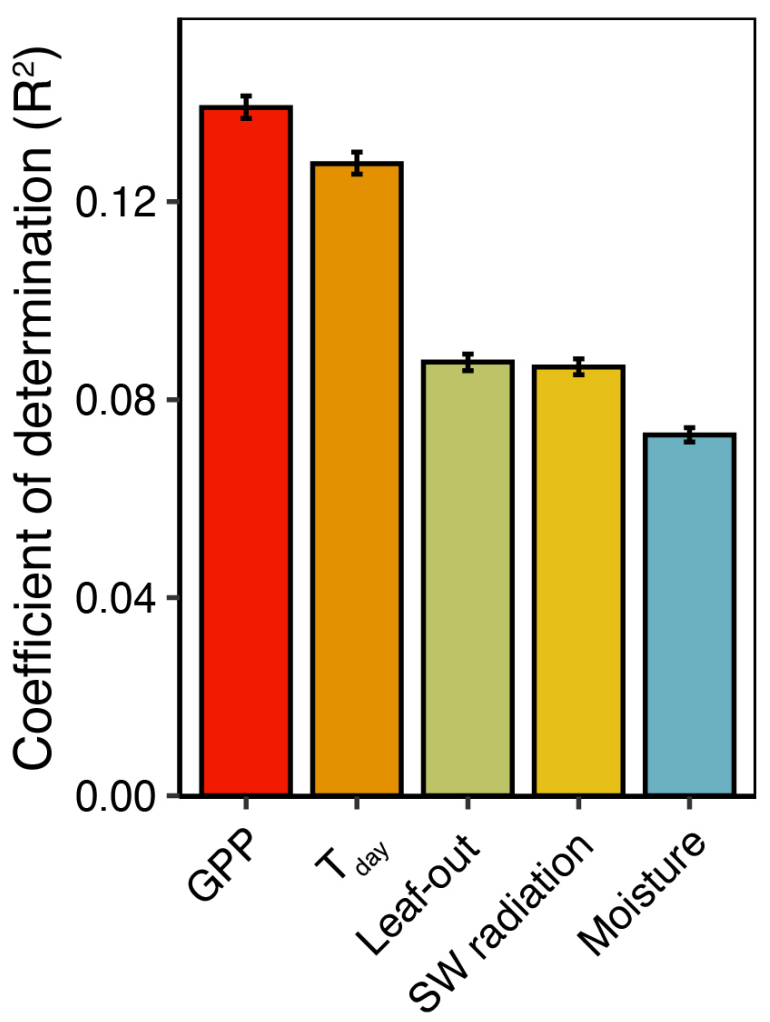

871

Extended Data Fig. 8. Coefficients of determination for the univariate effects of pre-solstice 874 productivity (GPP), pre-solstice temperature, spring leaf-out dates, pre-solstice short-wave

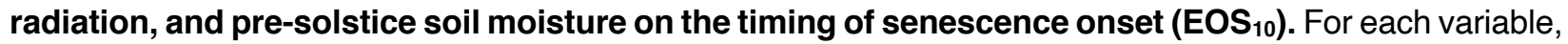
we ran univariate linear models, with GPP representing the cumulative productivity from leaf-out to June 21 (summer solstice), $\mathrm{T}_{\text {day }}$, SW radiation, and moisture representing the average day-time temperature, shortwave radiation, or soil moisture from March 20 to June 21, and leaf-out representing the spring leaf-out date

879 in the respective year. Models were run at the pixel-level and the mean coefficients of determination $( \pm 95 \%$

880

881

882 
a

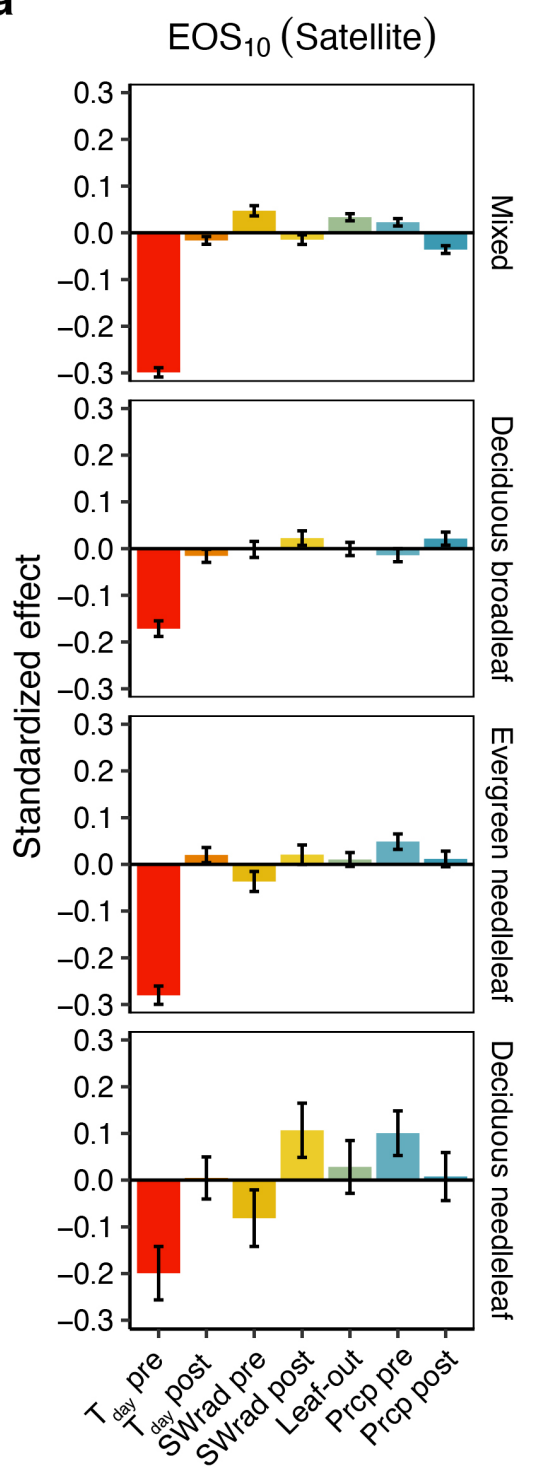

b
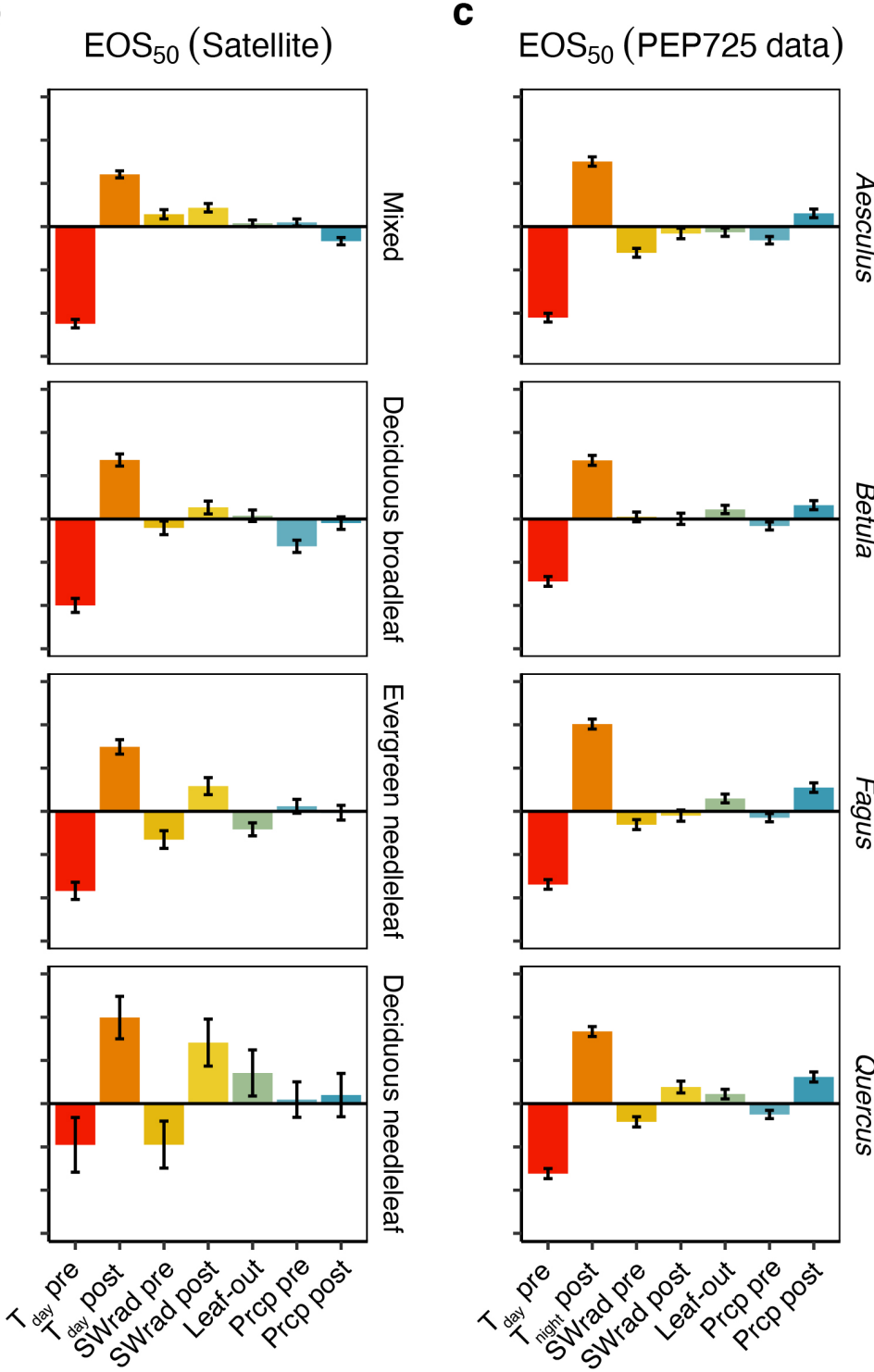

C

EOS $_{50}$ (PEP725 data)
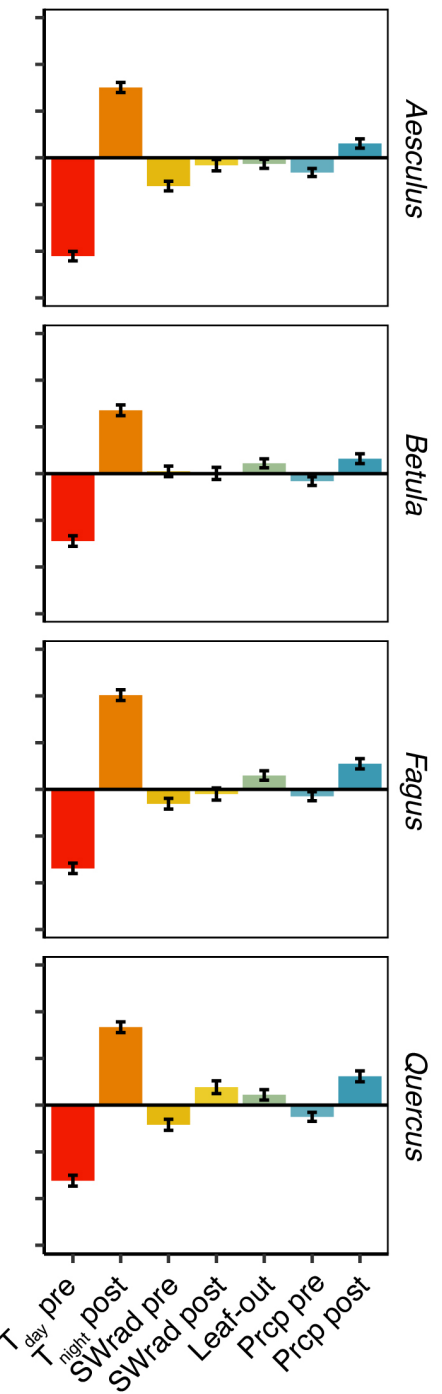

884

885

886

887

888

889

890

891

892

893

894
Extended Data Fig. 9. The effects of pre- and post-solstice temperature, radiation, precipitation and spring leaf-out dates on inter-annual variation in the timing of $\operatorname{EOS}_{10}(a)$ and $\operatorname{EOS}_{50}(b, c)$ for each forest type $(\mathbf{a}, \mathbf{b})$ and tree species (c). We ran linear models including mean day-time temperature $\left(T_{\text {day }}\right)$ and short-wave radiation (SWrad) and the sums of precipitation (prcp) from March 20 to June 21 (presolstice) and from June 22 to the mean EOS date of each time series (post-solstice) as well as spring leafout dates as predictor variables. Models were run at the pixel-level $(\mathbf{a}, \mathbf{b})$ or individual-level (c) and the mean effects ( $\pm 95 \%$ confidence intervals) are shown separately for mixed, deciduous broadleaf, evergreen needleleaf, and deciduous needleleaf forests for the satellite-based analyses and for the European species included in the PEP725 dataset. All variables were standardized to allow for effect size comparison. 


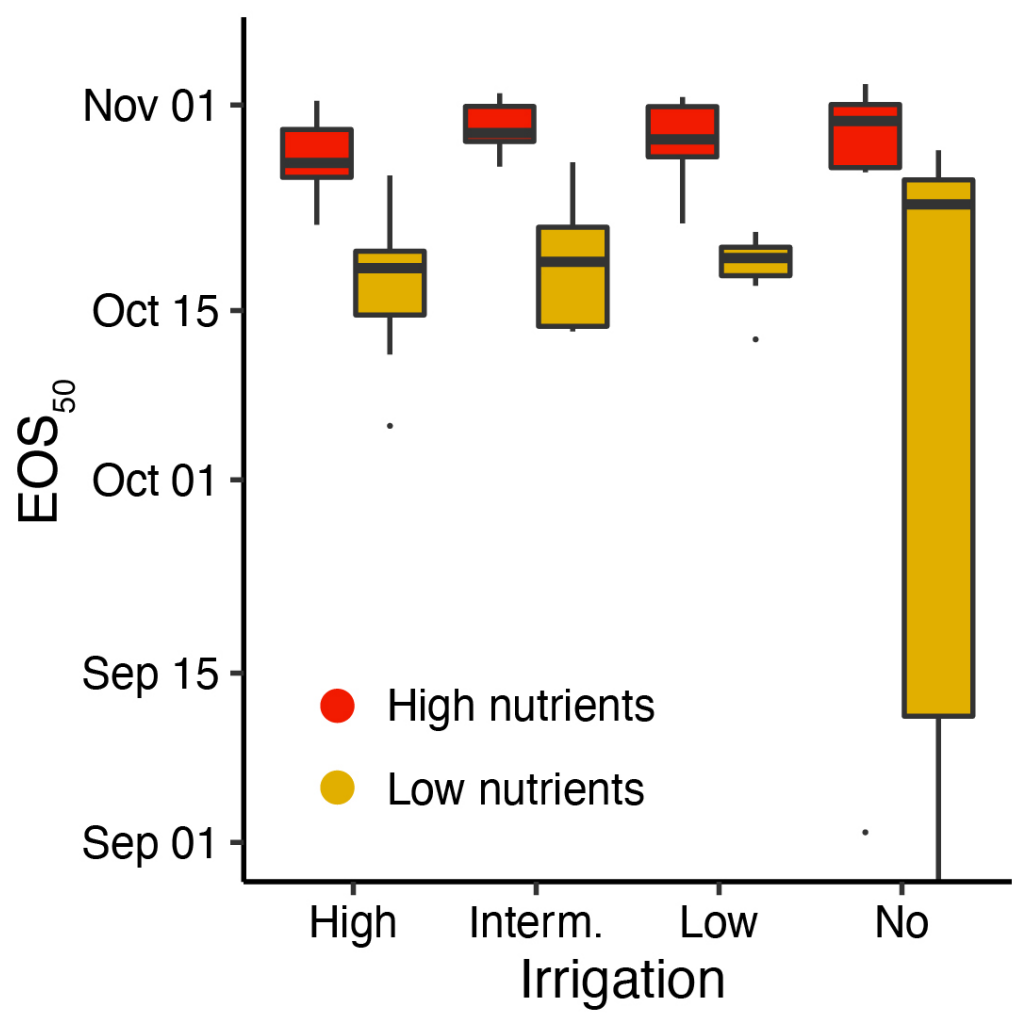

895

896

897

Extended Data Fig. 10. Results of the nutrient and drought experiment. $E \mathrm{~S}_{50}$ dates (day of $50 \%$

898 chlorophyll loss) for the eight treatment combinations, i.e., low and high soil nutrient status and no, low, intermediate and high irrigation. $P<0.01$ for the nutrient treatment and $P=0.17$ for the irrigation treatment.

900

901

902

903

904

905

906

907

908

909

910

911

912

913

914 


\section{Supplementary Files}

This is a list of supplementary files associated with this preprint. Click to download.

- ZohneretalSPSSupplement.pdf 